NASA/TP—2007-214480

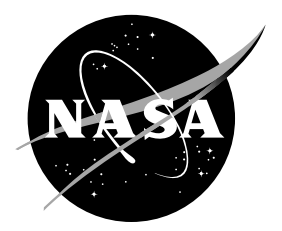

\title{
Compatibility Condition in Theory of Solid Mechanics (Elasticity, Structures, and Design Optimization)
}

Surya N. Patnaik

Ohio Aerospace Institute, Brook Park, Ohio

Shantaram S. Pai and Dale A. Hopkins

Glenn Research Center, Cleveland, Ohio 


\section{NASA STI Program . . . in Profile}

Since its founding, NASA has been dedicated to the advancement of aeronautics and space science. The NASA Scientific and Technical Information (STI) program plays a key part in helping NASA maintain this important role.

The NASA STI Program operates under the auspices of the Agency Chief Information Officer. It collects, organizes, provides for archiving, and disseminates NASA's STI. The NASA STI program provides access to the NASA Aeronautics and Space Database and its public interface, the NASA Technical Reports Server, thus providing one of the largest collections of aeronautical and space science STI in the world. Results are published in both non-NASA channels and by NASA in the NASA STI Report Series, which includes the following report types:

- TECHNICAL PUBLICATION. Reports of completed research or a major significant phase of research that present the results of NASA programs and include extensive data or theoretical analysis. Includes compilations of significant scientific and technical data and information deemed to be of continuing reference value. NASA counterpart of peer-reviewed formal professional papers but has less stringent limitations on manuscript length and extent of graphic presentations.

- TECHNICAL MEMORANDUM. Scientific and technical findings that are preliminary or of specialized interest, e.g., quick release reports, working papers, and bibliographies that contain minimal annotation. Does not contain extensive analysis.

- CONTRACTOR REPORT. Scientific and technical findings by NASA-sponsored contractors and grantees.
- CONFERENCE PUBLICATION. Collected papers from scientific and technical conferences, symposia, seminars, or other meetings sponsored or cosponsored by NASA.

- SPECIAL PUBLICATION. Scientific, technical, or historical information from NASA programs, projects, and missions, often concerned with subjects having substantial public interest.

- TECHNICAL TRANSLATION. Englishlanguage translations of foreign scientific and technical material pertinent to NASA's mission.

Specialized services also include creating custom thesauri, building customized databases, organizing and publishing research results.

For more information about the NASA STI program, see the following:

- Access the NASA STI program home page at http://www.sti.nasa.gov

- E-mail your question via the Internet to help@sti.nasa.gov

- Fax your question to the NASA STI Help Desk at 301-621-0134

- Telephone the NASA STI Help Desk at 301-621-0390

- Write to: NASA Center for AeroSpace Information (CASI) 7115 Standard Drive Hanover, MD 21076-1320 
NASA/TP—2007-214480

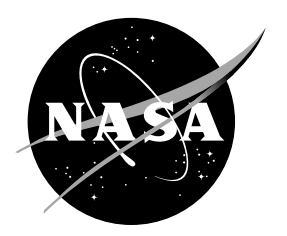

\section{Compatibility Condition in Theory of Solid Mechanics (Elasticity, Structures, and Design Optimization)}

Surya N. Patnaik

Ohio Aerospace Institute, Brook Park, Ohio

Shantaram S. Pai and Dale A. Hopkins

Glenn Research Center, Cleveland, Ohio

National Aeronautics and

Space Administration

Glenn Research Center

Cleveland, Ohio 44135 
This work was sponsored by the Fundamental Aeronautics Program at the NASA Glenn Research Center.

Level of Review: This material has been technically reviewed by an expert single reviewer.

Available from

NASA Center for Aerospace Information 7115 Standard Drive

Hanover, MD 21076-1320
National Technical Information Service 5285 Port Royal Road Springfield, VA 22161

Available electronically at http://gltrs.grc.nasa.gov 


\title{
Compatibility Condition in Theory of Solid Mechanics (Elasticity, Structures, and Design Optimization)
}

\author{
Surya N. Patnaik \\ Ohio Aerospace Institute \\ Brook Park, Ohio 44142 \\ Shantaram S. Pai and Dale A. Hopkins \\ National Aeronautics and Space Administration \\ Glenn Research Center \\ Cleveland, Ohio 44135
}

\section{Summary}

The strain formulation in elasticity and the compatibility condition in structural mechanics have neither been understood nor have they been utilized. This shortcoming prevented the formulation of a direct method to calculate stress and strain, which are currently obtained indirectly by differentiating the displacement. We have researched and understood the compatibility condition for linear problems in elasticity and in finite element structural analysis. This has led to the completion of the "method of force" with stress (or stress resultant) as the primary unknown. The method in elasticity is referred to as the completed Beltrami-Michell formulation (CBMF), and it is the integrated force method (IFM) in the finite element analysis. The dual integrated force method (IFMD) with displacement as the primary unknown has been formulated. Both the IFM and IFMD produce identical responses. The IFMD can utilize the equation solver of the traditional stiffness method. The variational derivation of the CBMF yielded the existing sets of elasticity equations along with the new boundary compatibility conditions, which were missed since the time of Saint-Venant, who formulated the field equations about 1860. The CBMF, which can be used to solve stress, displacement, and mixed boundary value problems, has eliminated the restriction of the classical method that was applicable only to stress boundary value problem. The IFM in structures produced high-fidelity response even with a modest finite element model. Because structural design is stress driven, the IFM has influenced it considerably. A fully utilized design method for strength and stiffness limitation has been developed via the IFM analysis tool, and its merits and limitations are discussed. The method has identified the singularity condition in structural optimization and furnished a strategy that alleviated the limitation and reduced substantially the computation time to reach the optimum solution. The CBMF and IFM tensorial approaches are robust formulations because both methods simultaneously emphasize the equilibrium equation and the compatibility condition. The vectorial displacement method emphasized the equilibrium, while the compatibility condition became the basis of the scalar stress-function approach. The tensorial approach can be transformed to obtain the vector and the scalar methods, but the reverse course cannot be followed. The tensorial approach outperformed other methods as expected. This report introduces the new concepts in elasticity, in finite element analysis, and in design optimization with numerical illustrations.

\subsection{Introduction}

The theory of solid mechanics remained incomplete for more than one century. The deficiency pertained to the strain formulation in elasticity and the compatibility condition in structural mechanics. Our research has alleviated the deficiency. It is now complete for linear elastic problems. A veteran researcher should not be surprised over a deficiency in the strain formulation because some of the formulae and equations of the solid mechanics discipline were not completed in the first attempt, but were perfected eventually. For example, perfecting the flexure formulae required more than one century between Galileo, Bernoulli, and Coulomb (ref. 1). Saint-Venant completed the shear stress formula that was initiated by Navier. Cauchy formulated the stress equilibrium equation that was also attempted by Navier in terms of displacement, but it contained only a single material constant instead of two (the rari-constant theory with one quarter for Poisson's ratio). Green (ref. 2) subsequently resolved the misconception. We have completed the strain formulation (ref. 3) that was initiated by Saint-Venant in 1860.

The compatibility condition (CC) is a significant ingredient in the theory of solid mechanics. Without a $\mathrm{CC}$, the discipline would degenerate into a few applied mathematics courses in the analysis of determinate systems. The CC makes solid mechanics a research discipline that is practiced in the academia and in major research institutions throughout the world. The seed of the compatibility condition is ingrained in Hooke's law, which forms the backbone of the theory of solid mechanics. The material law links stress $\{\sigma\}$ and strain $\{\varepsilon\}$ through the material matrix $[\kappa]$ :

$$
\{\sigma\}=[\kappa]\{\varepsilon\}
$$


Stress must satisfy the equilibrium equation (EE). Likewise, strain must satisfy the compatibility condition. That is, both stress and strain must simultaneously satisfy EE and $\mathrm{CC}$, respectively, because one (stress) can be considered as a scaled version of the other (strain) and vice versa. Because stress is a tensor quantity (with six components), its calculation requires an additional three compatibility conditions in addition to the three equilibrium equations. The tensorial nature does not allow the calculation of stress only from equilibrium considerations, and stress became an indeterminate quantity. The concept of indeterminacy is applicable to an elastic continuum as well as to a skeletal structure and a finite element model. A problem, either in continuum elasticity or in discrete structural analysis, is indeterminate if the number of stress unknowns $n$ exceed the displacements $m$. For example, a plane stress elasticity problem is one-degree indeterminate because there are three stresses, $n=3$, and two displacements, $m=2$, with a one-degree redundancy, $r=n-m=1$. Stress determination can be represented by a symbolic equation that is obtained by coupling the equilibrium equation and the compatibility condition:

$$
\begin{aligned}
{\left[\frac{\text { Equilibrium Equation }}{\text { Compatibility Condition }}\right]\{\text { Stress }\} } \\
=\left\{\frac{\text { Mechanical Load }}{\text { Initial Deformation }}\right\}
\end{aligned}
$$

Equation (2) provides both necessary and sufficient condition to calculate a high-fidelity stress state. Stress, calculated by any means or method, must be qualified through a compliance of equation (2); otherwise, such a stress state is liable to be incorrect. That is, the fidelity of stress determined via the stiffness method (ref. 4) has to be verified through a satisfaction of equation (2). Stress could not be calculated directly using equation (2) because the $\mathrm{CC}$ was not fully understood either in structures or in elasticity.

We have studied the CC in structures (ref. 5) as well as in elasticity (ref. 6). The theory of solid mechanics is completed by adding the new information on the $\mathrm{CC}$ to the existing set of equations. The pie diagram in figure 1 graphically depicts the stress formulation and the strain formulation. Cauchy (1789-1857) developed the stress formulation during the first quarter of the 19th century. It contained both the field equations and the boundary conditions for an elastic continuum. Decades later, Saint-Venant (1796-1886) provided the strain formulation (ref. 7) but only in the field of the continuum (shown as the third quarter in the pie chart). The boundary portion (marked as the shaded fourth quarter in the pie diagram), identified as the boundary compatibility condition (BCC), was missed until its formulation in 1986 (ref. 3). The compatibility concept in structures, developed through a "cut" and "close" gap technique, is not even

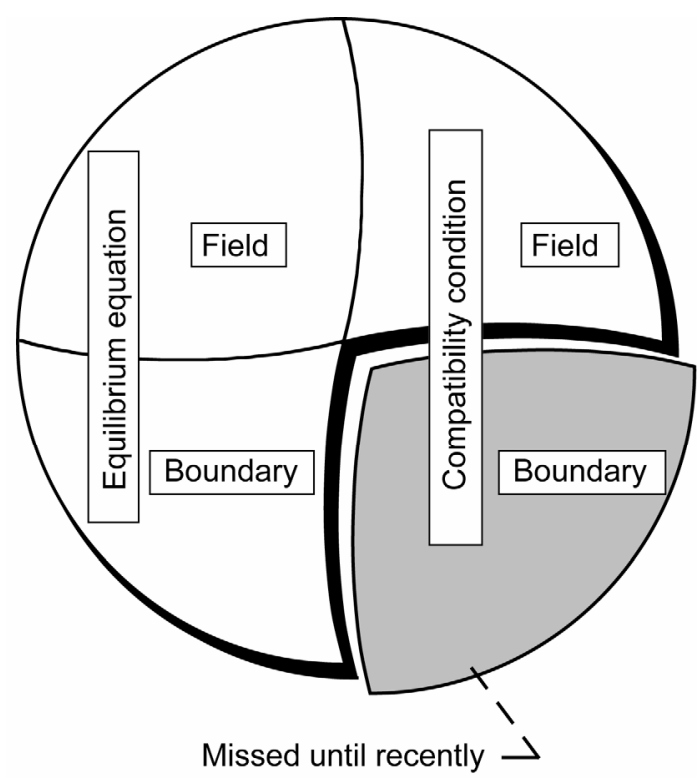

Figure 1.-Equilibrium equations and compatibility conditions in elasticity.

parallel to the available strain formulation in elasticity. The understanding and use of some aspects of the $\mathrm{CC}$, which remained immature, is depicted as the shaded fourth quarter in the pie chart.

Traditionally, solution to an indeterminate problem is obtained by utilizing the available information, contained within the unshaded three-quarter portion of the pie chart. Such approximate solution can be improved when information contained within the area of the entire pie diagram including the shaded area is utilized. The authors have formulated, verified, and utilized the additional information regarding the $\mathrm{CC}$ to solve problems. The available analysis methods for a solid mechanics problem are listed in table I. All analysis methods, including the flexibility method and the stiffness method, must satisfy both the EE and the CC; otherwise, the legitimacy of the predicted response can be questioned.

We have improved the direct stress calculation method and the associated variational formulation in the theory of solid mechanics. In elasticity, the new method (ref. 8) is referred to as the "completed Beltrami-Michell formulation" (CBMF), with stress as the primary unknown (see top row in table I). The CBMF is applicable to both stress and displacement boundary value problems. It has alleviated the limitation of the classical Beltrami-Michell formulation that was only applicable to the stress boundary value problem. The method in finite element structural analysis is called the integrated force method (IFM) with force as its primary unknown (ref. 9). The IFM is an improvement over the classical flexibility method because the redundant concept of 
TABLE I.-METHODS OF STRUCTURAL MECHANICS

AND ASSOCIATED VARIATIONAL FUNCTIONALS

\begin{tabular}{|c|c|c|c|c|c|}
\hline \multicolumn{2}{|c|}{ Method } & \multicolumn{2}{|c|}{ Primary variables } & \multirow{2}{*}{$\begin{array}{l}\text { Variational } \\
\text { functional }\end{array}$} & \multirow[t]{2}{*}{ Remarks } \\
\hline Elasticity & Structures & Elasticity & Structures & & \\
\hline $\begin{array}{l}\text { Completed } \\
\text { Beltrami-Michell } \\
\text { formulation } \\
\text { (CBMF) }\end{array}$ & $\begin{array}{l}\text { Integrated force } \\
\text { method (IFM) }\end{array}$ & Stresses & Forces & $\begin{array}{l}\text { IFM variational } \\
\text { functional }\end{array}$ & $\begin{array}{c}\text { Equilibrium and } \\
\text { compatibility } \\
\text { enforced }\end{array}$ \\
\hline Airy formulation & $\begin{array}{l}\text { Redundant } \\
\text { force method }\end{array}$ & Stress function & Redundants & $\begin{array}{c}\text { Complementary } \\
\text { energy }\end{array}$ & $\begin{array}{c}\text { Field } \\
\text { compatibility } \\
\text { enforced }\end{array}$ \\
\hline $\begin{array}{l}\text { Navier formula- } \\
\text { tion }\end{array}$ & $\begin{array}{l}\text { Stiffness } \\
\text { method }\end{array}$ & Displacements & Deflections & Potential energy & $\begin{array}{c}\text { Boundary } \\
\text { compatibility } \\
\text { noncompliant }\end{array}$ \\
\hline Hybrid method & $\begin{array}{l}\text { Reissner } \\
\text { method }\end{array}$ & $\begin{array}{c}\text { Stresses and } \\
\text { displacements }\end{array}$ & $\begin{array}{l}\text { Forces and } \\
\text { deflections }\end{array}$ & $\begin{array}{l}\text { Reissner func- } \\
\text { tional }\end{array}$ & $\begin{array}{c}\text { Boundary } \\
\text { compatibility } \\
\text { noncompliant }\end{array}$ \\
\hline Total formulation & $\begin{array}{l}\text { Washizu } \\
\text { method }\end{array}$ & $\begin{array}{c}\text { Stresses, } \\
\text { strains, and } \\
\text { displacements }\end{array}$ & $\begin{array}{l}\text { Forces, defor- } \\
\text { mations, } \\
\text { and deflections }\end{array}$ & $\begin{array}{l}\text { Washizu func- } \\
\text { tional }\end{array}$ & $\begin{array}{l}\text { Boundary } \\
\text { compatibility } \\
\text { noncompliant }\end{array}$ \\
\hline
\end{tabular}

"cut" and "close" gap is not used, and it is applicable to dynamic analysis (ref. 10). Realizing the prominence of the stiffness method, a dual formulation referred to as the dual integrated force method (IFMD) (ref. 11) has been obtained. The equation structure of the IFMD and the stiffness method are similar in form and size, only the numerical values differ in the matrix $[D]$ of the IFMD $([D]\{X\}=\{P\})$ and $[K]$ of stiffness method $([K]\{X\}=\{P\})$; here $\{X\}$ and $\{P\}$ are displacement and load, respectively. The variational formulation of the new methods is referred to as the IFM variational functional $\left(\pi_{S}\right)$ (ref. 3 ). The stationary condition of the new functional yields both stress and strain formulations, new boundary compatibility conditions, and the displacement continuity conditions.

The CBMF and IFM methods listed in the first row in table I can be considered as tensorial approaches in analysis because their primary unknown is either stress or internal force, which is a stress resultant. The tensor approach emphasizes both equilibrium and compatibility concepts. The stiffness and Navier's displacement methods are vectorial approaches, which emphasize the equilibrium equation. The stress function approach is the scalar method, and the compatibility condition is its basis. The tensor approach can be specialized to obtain the vector and scalar methods, but the reverse course cannot be followed. For example, the equations of the new methods (IFM and CBMF) can be specialized to obtain the existing formulations of solid mechanics, such as Airy's formulation, the stiffness method, the hybrid method, and the total formulation, as listed in table I. Navier's formulation cannot be transformed to obtain the CBMF in elasticity. Likewise, the IFM variational functional $\pi_{s}$ can be specialized to obtain the potential energy and complementary energy functionals, but the reverse course cannot be followed.

Because structural design is stress driven, the IFM has become a useful tool in traditional fully stressed design as well as in design optimization. As it turns out, the IFM was devised to study the feasibility of full stress design in an indeterminate truss (ref. 12). An indeterminate truss cannot be fully stressed (ref. 13). This paper introduces the new compatibility concepts in elasticity, finite element analysis, and design optimization. This is followed by a conclusion section. A list of symbols and acronyms used in this report is given in appendix A to aid the reader, and appendix B contains a bibliography on the method of force for an in-depth study by an interested reader.

\subsection{Elasticity}

The material law is the foundation upon which the theory of solid mechanics is built. This stress $(\sigma)$-strain $(\varepsilon)$ law (eq. (1)) was formulated and interpreted by Hooke (16351703), Young (1773-1829), Poisson (1781-1840), and others. The material law contained the essence of structural analysis. The constraint imposed on stress became the stress formulation, or the EE. Likewise, the condition on the strain became the strain formulation, or the CC. The stress and strain formulations along with the material matrix $[\kappa]$ given in equation (1) are sufficient for the determination of the stress state in an elastic continuum. The stress formulation is credited to Cauchy (1789-1857), who has formulated the field equation and the boundary condition, also known as the 
traction condition. Saint-Venant (1797-1886) developed the strain formulation, and it was rederived by Sokolnikoff in its popular form (ref. 7). It was a general belief that the fundamental elasticity relations were known for over a century. The thrust, therefore, was to develop approximate techniques because a closed-form solution can seldom be generated for the vast majority of the elasticity problems. In elasticity, Airy's stress function technique (ref. 14) and Ritz's displacement formulation (ref. 15) are two popular methods. Airy's method is based on the compatibility concept, whereas Ritz's method utilized the equilibrium equation. Their equivalents in the theory of structure and finite element analysis became the flexibility and the stiffness methods, respectively.

We now discuss the incompleteness in the strain formulation, which is quite straightforward to comprehend. Consider Cauchy's stress formulation. It is complete because the treatment included the field as well as the boundary of an elastic continuum. In the field,

$$
\tau_{i j, j}+b_{i}=0
$$

where $\tau_{i j}$ is the stress tensor and $b_{i}$ is body force. On the boundary,

$$
\tau_{i j} n_{j}=p_{i}
$$

where $n_{j}$ is the direction cosine of the outward normal and $p_{i}$ is a traction component.

Likewise, the strain formulation should have contained conditions in the field as well as on the boundary. However, it was formulated only in the field. The strain formulation in the field $f_{c c}$ for a plane elasticity problem can be written as

$$
f_{c c}=\frac{\partial^{2} \varepsilon_{y}}{\partial x^{2}}+\frac{\partial^{2} \varepsilon_{x}}{\partial y^{2}}-\frac{\partial^{2} \gamma_{x y}}{\partial x \partial y}=0
$$

where $\varepsilon_{x}, \varepsilon_{y}$, and $\gamma_{x y}=2 \varepsilon_{x y}$ are strain components. Comparing stress and strain formulations we observe

(1) Field equations are available for both stress and strain formulations (eqs. (3) and (5)).

(2) The boundary condition is available only for the stress formulation (eq. (4)) but not for the strain formulation.

The strain formulation remained incomplete since the time of Saint-Venant. That is, the theory of elasticity was incomplete since 1860 . Because of this deficiency, the classical Beltrami-Michell formulation could not be used to solve an elastic continuum with a displacement boundary condition. The strain formulation on the boundary has recently been completed (ref. 3). The boundary compatibility condition (BCC) for the plane stress problem can be written as

$$
\mathrm{BCC}=\left(\frac{\partial \varepsilon_{y}}{\partial x}-\frac{\partial \varepsilon_{x y}}{\partial y}\right) n_{x}+\left(\frac{\partial \varepsilon_{x}}{\partial y}-\frac{\partial \varepsilon_{x y}}{\partial x}\right) n_{y}=0
$$

where $n_{x}$ and $n_{y}$ are the direction cosines of the outward normal. With the BCC, the stress and strain formulations given above have parallel forms, each containing field equations (eqs. (3) and (5)), and boundary conditions (eqs. (4) and (6)). The addition of the new BCC has completed the Beltrami-Michell formulation in elasticity. The completed method can be used to solve all three types of boundary value problems in elasticity: the stress boundary value problem, the displacement boundary value problem, and the mixed boundary value problem. The triviality of the known strain formulation in the field $\left(f_{c c}(u, v)=\mathscr{S}(u, v)-\mathscr{f}(u, v)=0\right.$, see eq. (5)) justified Navier's method, which is obtained by rewriting Cauchy's equilibrium equation in the displacement variables. Navier's method can satisfy the compatibility condition in the field but not on the boundary because the new BCC is not automatically satisfied when expressed in displacements $(u$ and $v)$ :

$$
\begin{aligned}
\mathrm{BCC} & =\left[\frac{\partial^{2} v}{\partial x \partial y}-\frac{1}{2} \frac{\partial}{\partial y}\left(\frac{\partial u}{\partial y}+\frac{\partial v}{\partial x}\right)\right] n_{x} \\
& +\left[\frac{\partial^{2} u}{\partial x \partial y}-\frac{1}{2} \frac{\partial}{\partial x}\left(\frac{\partial v}{\partial x}+\frac{\partial u}{\partial y}\right)\right] n_{y}=0
\end{aligned}
$$

The consequence of this noncompliance has to be investigated. The usefulness of the BCC is further investigated considering the CBMF in polar coordinates because this is a popular topic in the theory of elasticity.

\subsection{CBMF in Polar Coordinates}

The strain formulation (that includes the field and boundary $\mathrm{CCs}$ ) is required in the theory of elasticity because both stress and strain are tensor quantities. A variational approach has to be followed to derive the strain formulation, along with other equations for a two-dimensional continuum in polar coordinates because the boundary compatibility condition cannot be generated from a nonvariational direct approach. The stationary condition of the IFM functional yields both the stress and the strain formulations as well as the displacement continuity condition - or all equations of the CBMF. Consider a plane elastic continuum with uniform thickness $t$ in the $(r, \theta)$ polar coordinate system, as shown in figure 2. It is made of an isotropic material with Young's modulus $E$ and Poisson's ratio v. It is subjected to external load $\left(\bar{P}_{r}\right.$ and $\left.\bar{P}_{\theta}\right)$ along the boundary segment $\left(\ell_{1}\right)$. On the remainder of the boundary $\left(\ell_{2}\right)$, the displacements are 

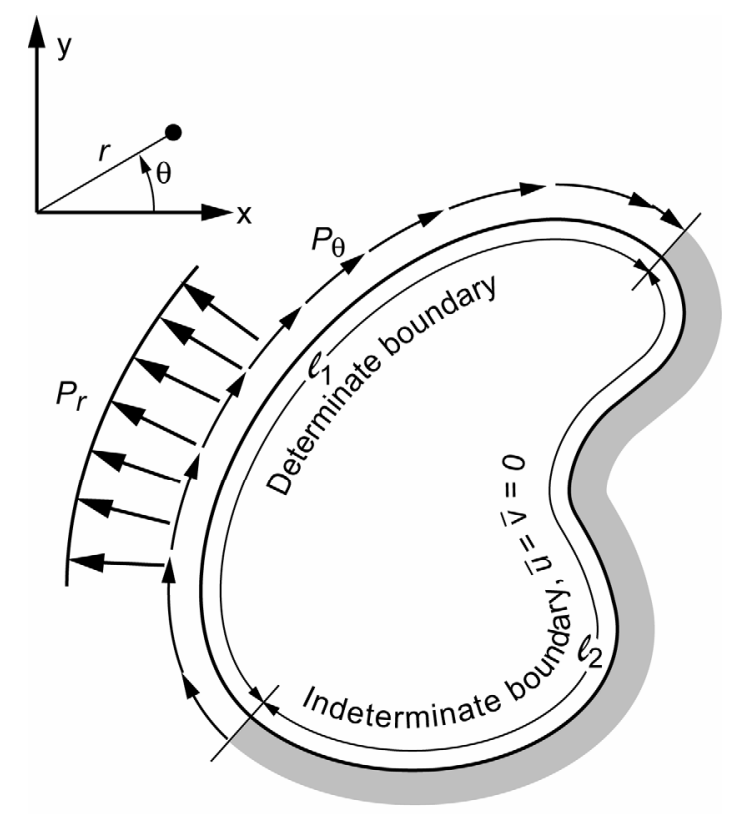

Figure 2.-Two-dimensional elastic continuum in polar coordinates with loads $P_{r}$ and $P_{\theta}$ and prescribed displacements $\bar{u}$ and $\bar{v}$.

restrained $(\bar{u}=0$ and $\bar{v}=0)$. Three stresses $\left(\sigma_{r}, \sigma_{\theta}, \tau_{r \theta}=\tau\right)$ and two displacements $(u$ and $v)$ are the unknowns of the problem. Three strains $\left(\varepsilon_{r}, \varepsilon_{\theta}, \gamma_{r \theta}=\gamma\right)$ can be calculated by scaling the stresses using Hooke's law. The stationary condition of the functional $\pi_{s}$ of the IFM (ref. 6) with respect to displacement and stress function yields both the stress and strain formulations as well as the displacement continuity condition. The functional has three terms $A, B$, and $W$ as follows:

$$
\pi_{s}=A+B-W
$$

The term $A(\sigma, u)$ represents the strain energy, expressed in terms of stress $\sigma$ and displacement $u$. The term $B(\varepsilon, \varphi)$ is the complementary strain energy written in strain $\varepsilon$ and the stress function $\varphi$. The potential of the work done is $W$. The definitions of $A, B$, and $W$ follow:

$$
\begin{aligned}
A & =\oiint_{D}\left(\frac{1}{\sigma_{r} \partial u}+\frac{2}{\partial r}+\frac{\tau \partial u}{r \partial \theta}+\sigma_{\theta} u\right. \\
& \left.+\frac{4}{\partial r}+\frac{5}{r \partial \theta}-\frac{\sigma_{\theta} \partial v}{r}\right) t r \mathrm{~d} r \mathrm{~d} \theta
\end{aligned}
$$

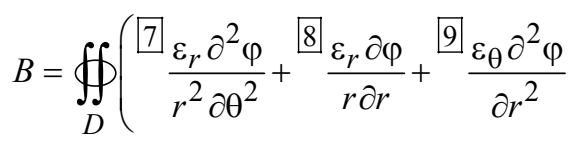

$$
\begin{aligned}
& \left.+\frac{\gamma \partial \varphi}{r^{2} \partial \theta}-\frac{11}{r \partial r \partial \theta}\right) t r \mathrm{~d} r \mathrm{~d} \theta \\
& W=\left[t \oint_{\ell_{1}}\left(\frac{12}{u \bar{P}_{r}+13} v \bar{P}_{\theta}\right) \mathrm{d} \ell_{1}+\oint_{\ell_{2}}\left(\frac{14}{u} R_{r}+{ }^{15} R_{\theta}\right) \mathrm{d} \ell_{2}\right. \\
& \left.+\oiint_{D}\left(b_{r} u+b_{\theta} v\right)(r \mathrm{~d} r \mathrm{~d} \theta)\right]
\end{aligned}
$$

The domain $(D)$ has boundary $\ell=\ell_{1}+\ell_{2}$. Body forces are $\left(b_{r}\right.$ and $\left.b_{\theta}\right)$. Along the boundary segment $\ell_{1}$, loads $\bar{P}_{r}$ and $\bar{P}_{\theta}$ are prescribed, whereas displacements $(u$ and $v)$ are set free. The segment $\ell_{2}$ has prescribed displacements $\bar{u}$ and $\bar{v}$ that can induce reactions $R_{r}$ and $R_{\theta}$. The derivation sets the uniform thickness to unity $(t=1)$ without any consequence. Body force potential $V$ is defined as

$$
b_{r}=\frac{\partial V}{\partial r} \text { and } b_{r}=\frac{\partial V}{r \partial \theta}
$$

The stress function $\varphi$ is defined as

$$
\begin{gathered}
\sigma_{r}=\frac{\partial \varphi}{r \partial r}+\frac{\partial^{2} \varphi}{r^{2} \partial \theta^{2}}-V \\
\sigma_{\theta}=\frac{\partial^{2} \varphi}{\partial r^{2}}-V \\
\tau=-\frac{\partial}{\partial r}\left(\frac{\partial \varphi}{r \partial \theta}\right)
\end{gathered}
$$

Details of the derivation are given in reference 6 and are not repeated here. The final expression of the stationary condition of the functional with respect to displacement and stress function has the following form: 


$$
\begin{aligned}
\delta \pi_{S} & =\oiint_{D}[(\text { field EE }) \delta\{u\}+(\text { field CC }) \delta \varphi] \mathrm{d} s \\
& +\oint_{\ell_{1}}[(\text { boundary EE }) \delta\{u\}] \mathrm{d} \ell \\
& +\oint_{\ell}[(\text { boundary CC }) \delta \varphi] \mathrm{d} \ell \\
& +\oint_{\ell_{2}}(\text { continuity condition }) \delta\{\text { reaction }\} \mathrm{d} \ell=0
\end{aligned}
$$

The field EE and field CC are the coefficients of the variational displacement and stress function, respectively, in the surface integral terms of the functional in equation (12). Likewise the boundary EE (or traction condition) and the new boundary $\mathrm{CC}$ are the coefficients of the variational displacement and stress function, respectively, in the line integral terms. The continuity conditions are the coefficients of the variational reactions. The $\mathrm{CC}$ becomes relevant when the variational stress function is nonzero $(\delta \varphi \neq 0)$ or the domain is indeterminate. The CC should not be enforced on a determinate domain with $\delta \varphi=0$.

The field equations and boundary conditions of the CBMF recovered from the stationary condition of the variational functional are listed below. The CCs recovered in equation (12) are in strains, which are transformed into stress using Hooke's law.

\section{Completed Beltrami-Michell Formulation in Polar Coordinates:}

Field equilibrium equations:

$$
\begin{gathered}
\frac{\partial \sigma_{r}}{\partial r}+\frac{1}{r} \frac{\partial \tau}{\partial \theta}+\frac{\left(\sigma_{r}-\sigma_{\theta}\right)}{r}+b_{r}=0 \\
\frac{\partial \tau}{\partial r}+\frac{1}{r} \frac{\partial \sigma_{\theta}}{\partial \theta}+\frac{2 \tau}{r}+b_{\theta}=0
\end{gathered}
$$

Boundary traction conditions:

$$
\begin{aligned}
& n_{r} \sigma_{r}+n_{\theta} \tau=\bar{P}_{r} \\
& n_{r} \tau+n_{\theta} \sigma_{\theta}=\bar{P}_{\theta}
\end{aligned}
$$

Field compatibility condition:

$$
\begin{aligned}
\left(\frac{1}{E}\right)\left[\frac{1}{r^{2}} \frac{\partial^{2} \sigma_{r}}{\partial \theta^{2}}-v \frac{\partial^{2} \sigma_{r}}{\partial r^{2}}-\frac{(1+2 v)}{r} \frac{\partial \sigma_{r}}{\partial r}+\frac{\partial^{2} \sigma_{\theta}}{\partial r^{2}}\right. \\
-\frac{v}{r^{2}} \frac{\partial^{2} \sigma_{\theta}}{\partial \theta^{2}}+\frac{(1+2 v)}{r} \frac{\partial \sigma_{\theta}}{\partial r}-\frac{(1+v)}{r} \frac{\partial^{2} \tau}{\partial r \partial \theta} \\
\left.-\frac{(1+v)}{r^{2}} \frac{\partial \tau}{\partial r}\right]=0
\end{aligned}
$$

Boundary compatibility condition:

$$
\begin{aligned}
& {\left[\frac{\partial}{\partial r}\left(\sigma_{\theta}-v \sigma_{r}\right)-\frac{(1+v)}{r}\left(\frac{\partial \tau}{\partial \theta}-\sigma_{\theta}+\sigma_{r}\right)\right] n_{r}} \\
& +\left[(1+v) \frac{\partial \tau}{\partial r}-(1+v) \frac{\partial \sigma_{\theta}}{\partial \theta}+\frac{2(1+v)}{r} \tau\right] n_{\theta}=0
\end{aligned}
$$

The kinematics boundary conditions are

$$
\begin{aligned}
& u-\bar{u}=0 \\
& v-\bar{v}=0
\end{aligned}
$$

where

$$
\begin{array}{ll}
r \text { and } \theta & \text { polar coordinates } \\
\sigma_{r}, \sigma_{\theta} \text {, and } \tau & \text { stress components } \\
\varepsilon_{r}, \varepsilon_{\theta} \text {, and } \gamma & \text { strain components } \\
u \text { and } v & \text { displacements } \\
b_{r} \text { and } b_{\theta} & \text { body forces } \\
\overline{P_{r}} \text { and } \overline{P_{\theta}} & \text { tractions applied along boundary segment } \ell_{1} \\
\bar{u} \text { and } \bar{v} & \text { initial displacements along boundary segment } \\
& \ell_{2} \\
n_{r} \text { and } n_{\theta} & \text { direction cosines of the outward normal }
\end{array}
$$

\subsubsection{Verification of boundary compatibility condition.-} Green's theorem is used for a quick verification of the new BCC. The BCC is inserted in the line integral coefficient to recover the well-known field CC in the surface integral term. The integral theorem in polar coordinates can be written as

$$
\oiiint\left(\frac{1}{r} \frac{\partial}{\partial r}\left(r G_{r}\right)-\frac{\partial G_{\theta}}{r \partial \theta}\right) \mathrm{d} s=\oint\left(G_{r} n_{r}+G_{\theta} n_{\theta}\right) \mathrm{d} \ell
$$


where $G_{r}$ and $G_{\theta}$ are the coefficients of direction cosines $n_{r}$ and $n_{\theta}$, respectively. The coefficients are defined for the CBMF as

$$
\begin{gathered}
G_{r}=\frac{\varepsilon_{r}}{r}-\frac{\partial\left(r \varepsilon_{\theta}\right)}{r \partial r}+\frac{\partial \gamma}{2 r \partial \theta} \\
G_{\theta}=\frac{\partial \varepsilon_{r}}{r \partial \theta}-\frac{\partial \gamma}{2 \partial r}-\frac{\gamma}{r}
\end{gathered}
$$

The surface integral terms are recovered as

$$
\begin{gathered}
\frac{\partial\left(r G_{r}\right)}{r \partial r}=\left(\frac{\partial \varepsilon_{r}}{r \partial r}\right)-\left(\frac{2 \partial \varepsilon_{\theta}}{r \partial r}+\frac{\partial^{2} \varepsilon_{\theta}}{\partial r^{2}}\right)+\frac{\partial^{2} \gamma}{2 r \partial r \partial \theta} \quad \text { (18d) } \\
\frac{\partial G_{\theta}}{r \partial \theta}=\frac{\partial^{2} \varepsilon_{r}}{r^{2} \partial \theta^{2}}-\frac{\partial^{2} \gamma}{2 r \partial r \partial \theta}-\frac{\partial \gamma}{r^{2} \partial \theta} \quad(18 \mathrm{e}) \\
\oiint\left[\frac{\partial\left(r G_{r}\right)}{r \partial r}-\frac{\partial G_{\theta}}{r \partial \theta}\right] \mathrm{d} s \\
=-\oiint\left(\frac{\partial^{2} \varepsilon_{r}}{r^{2} \partial \theta^{2}}-\frac{\partial \varepsilon_{r}}{r \partial r}+\frac{\partial^{2} \varepsilon_{\theta}}{\partial r^{2}}+\frac{2 \partial \varepsilon_{\theta}}{r \partial r}-\frac{\partial \gamma}{r^{2} \partial \theta}-\frac{\partial^{2} \gamma}{r \partial r \partial \theta}\right) \mathrm{d} s
\end{gathered}
$$

The right-hand coefficient in equation (18f) is the field CC. The compatibility concept applies to the field as well as to the boundary. The analytical form of the compatibility expression changes in compliance with the domain and the boundary. The same interpretation is true for Cauchy's field EEs.

The BCC written in displacements is not a trivial condition:

$$
\begin{aligned}
& \left(\frac{\partial^{2} u}{\partial \theta^{2}}-r \frac{\partial^{2} v}{\partial r \partial \theta}-\frac{\partial v}{\partial \theta}\right) n_{r} \\
& +\left(r \frac{\partial^{2} u}{\partial r \partial \theta}+v-r^{2} \frac{\partial^{2} v}{\partial r^{2}}-r \frac{\partial v}{\partial r}-r \frac{\partial u}{\partial \theta}\right) n_{\theta}=0
\end{aligned}
$$

The field $\mathrm{CC}$ is a second-order differential equation in strains, while the boundary counterpart is a first-order equation. This characteristic is applicable to the stress formulation. The field EEs are first-order equations in stress, while the boundary (or traction) conditions are algebraic equations. The $\mathrm{BCC}$ is not a continuity condition in displacement. The BCC is an independent condition. It forms a new elasticity expression that was missed since the time of Saint-Venant.

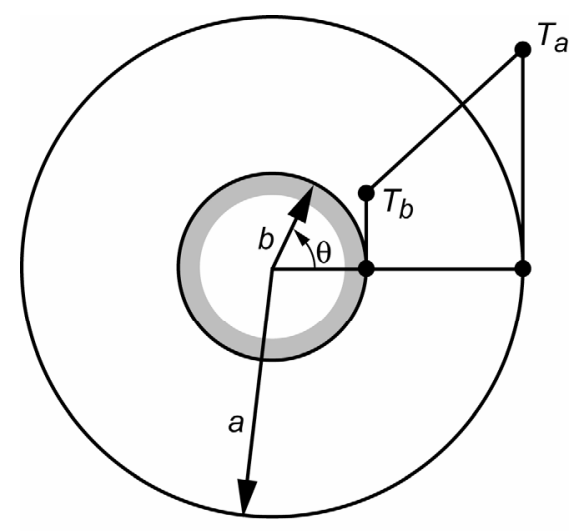

Figure 3.-Annular plate subjected to linear temperature distribution.

2.1.2 Illustrative example: annular plate subjected to thermal load.-We will now illustrate the CBMF calculation strategy and the use of the BCC through the solution of a radially symmetrical annular plate with mixed boundary conditions for thermal load. Consider a plate made of an isotropic material with Young's modulus $E$ and Poisson's ratio $v$. It has thickness $t$ (considered unity) with outer and inner radii of $a$ and $b$, respectively, as shown in figure 3 . The inner boundary is fully restrained while the outer boundary is free to expand. The annular plate is subjected to a temperature distribution:

$$
T=T_{b}+\frac{\left(T_{a}-T_{b}\right)}{(a-b)}(r-b)
$$

The temperature has a linear variation with values $T_{a}$ and $T_{b}$ at $r=a$ and $r=b$, respectively. The coefficient of thermal expansion is $\alpha$. The CBMF equations for the annular plate subjected to a thermal load are given below:

\section{Equilibrium equations}

Field:

$$
\frac{\partial \sigma_{r}}{\partial r}+\frac{\left(\sigma_{r}-\sigma_{\theta}\right)}{r}=0
$$

Traction or boundary:

$$
\sigma_{r}=0 \text { at } r=a
$$

\section{Compatibility conditions}

Field:

$$
\frac{\partial}{\partial r}\left(\sigma_{\theta}-v \sigma_{r}\right)+\frac{(1+v)}{r}\left(\sigma_{\theta}-\sigma_{r}\right)=-\alpha E \frac{\mathrm{d} T}{\mathrm{~d} r}
$$


Boundary:

$$
\sigma_{\theta}-v \sigma_{r}=-\alpha E T \text { at } r=b
$$

\section{Displacement boundary condition}

$$
u=0 \text { at } r=b
$$

The temperature term is contained explicitly in both field and boundary CCs (see eqs. (22a) and (22b)). The EE and $\mathrm{CC}$ in the field are rearranged to obtain the following two simple working equations:

$$
\begin{gathered}
\frac{\mathrm{d}}{\mathrm{d} r}\left(\sigma_{r}+\sigma_{\theta}\right)=-\alpha E \frac{\mathrm{d} T}{\mathrm{~d} r} \\
\frac{\mathrm{d} \sigma_{r}}{\mathrm{~d} r}+\frac{\left(\sigma_{r}-\sigma_{\theta}\right)}{r}=0
\end{gathered}
$$

The CBMF solution strategy has two distinct steps. First, the stress state is calculated from the field $\mathrm{EE}$ and $\mathrm{CC}$ given by equations (24a) and (24b), for the boundary conditions given by equations (21b) and (22b). The displacement boundary condition (given by eq. (23)) is not used to calculate the stress state. Pure displacement cannot induce stress. Only the derivative of displacement, which is strain, produces stress. The stress solution follows:

$$
\begin{aligned}
\sigma_{r}=\left\{\frac{E \alpha(a-r)}{3 r^{2}(a-b)\left[a^{2}+b^{2}+v\left(a^{2}-b^{2}\right)\right]}\right\} \\
\times\left(T_{a}\left\{\begin{array}{l}
r^{2}\left[a^{2}+b^{2}+v\left(a^{2}-b^{2}\right)\right] \\
+r b^{2}[a(1-v)-b(2-v)] \\
+a b^{2}[a(1-v)-b(2-v)]
\end{array}\right\}\right. \\
+T_{b}\left\{\begin{array}{l}
+r b^{2}[a(2+v)-b(1+v] \\
-r^{2}\left[a^{2}+b^{2}+v\left(a^{2}-b^{2}\right)\right] \\
+a b^{2}[-b(1+v)+a(2+v)]
\end{array}\right\}
\end{aligned}
$$

$\sigma_{\theta}=\left\{\frac{E \alpha}{3 r^{2}(b-a)\left[a^{2}+b^{2}+v\left(a^{2}-b^{2}\right)\right]}\right\}$

$$
\begin{aligned}
& \times\left(\begin{array}{l}
2 r^{3}\left[a^{2}+b^{2}+v\left(a^{2}-b^{2}\right)\right] \\
-r^{2}\left[a^{3}+2 b^{3}+v\left(a^{3}-b^{3}\right)\right] \\
+a^{2} b^{2}[a(1-v)-b(2-v)]
\end{array}\right\} \\
& \left.+T_{b}\left\{\begin{array}{l}
+r^{2}\left[a^{3}-b^{3}+3 a b^{2}+v\left(a^{3}-b^{3}\right)\right] \\
+a^{2} b^{2}[a(2+v)-b(1+v)]
\end{array}\right\}\right)
\end{aligned}
$$

Next, the displacement function is back-calculated by integrating the stress. The displacement boundary condition $u=0$ at $r=b$ is used to evaluate the integration constant. The displacement function is

$$
\begin{aligned}
u & =\left\{\begin{array}{c}
\alpha(1+v)(b-r) \\
3 r^{2}(b-a)\left[a^{2}+b^{2}+v\left(a^{2}-b^{2}\right)\right]
\end{array}\right\} \\
\times & \left(\begin{array}{l}
r^{2}\left[a^{2}+b^{2}+v\left(a^{2}-b^{2}\right)\right] \\
+r a^{2}[a-2 b-v(a-b)] \\
+a^{2} b[a(1-v)-b(2-v)]
\end{array}\right\} \\
+ & T_{b}\left\{\begin{array}{l}
+r a^{2}[2 a-b+v(a-b)] \\
-r^{2}\left[a^{2}+b^{2}+v\left(a^{2}-b^{2}\right)\right] \\
+a^{2} b[a(2+v)-b(1+v)]
\end{array}\right\}
\end{aligned}
$$

The numerical values of the response parameters for $T_{a}=$ $100{ }^{\circ} \mathrm{C}, T_{b}=50{ }^{\circ} \mathrm{C}$, and $\alpha=12 \times 10^{-6} /{ }^{\circ} \mathrm{C}$ are

(1) at $r=a: \sigma_{r}=0 \mathrm{ksi}, \sigma_{\theta}=-17.5 \mathrm{ksi}$, and $u=0.012 \mathrm{in}$.

(2) at $r=b: \sigma_{r}=14.2 \mathrm{ksi}, \sigma_{\theta}=-13.7 \mathrm{ksi}$, and $u=0 \mathrm{in}$. 
The sum of the stresses $\sigma_{r}+\sigma_{\theta}=18508-1800 r$ has a linear variation with respect to the $r$-coordinate because of a similar distribution of temperature (see eq. (20)). The solution steps in the CBMF are discussed in appendix $\mathrm{C}$.

\subsection{Treatment of Boundary Compatibility in Na- vier's Displacement Method}

The displacement method of Navier is universally accepted, and it has been applied to solve many problems. It has field equations and an appropriate set of boundary conditions. Adding an extra BCC (see eq. (19) for example) may appear to hamper the method with an excessive number of boundary conditions for which no analytical solution can be generated. This observation is an aberration but has no serious implication. We discuss the BCC by solving a plate flexure problem because this is a popular example and its solution occupies a good portion of textbooks (ref. 16). The rectangular plate is made of an isotropic material with Young's modulus $E$ and Poisson's ratio v, thickness $t$, and spans of $2 a$ and $2 c$ along $x$ - and $y$-coordinate directions, respectively, as shown in figure 4 . It is subjected to a distributed transverse load $q$ with a sinusoidal variation along the $x$ coordinate direction, while being uniform along the $y$ direction as

$$
q=q_{0} \cos (\pi x / 2 a)
$$

The plate is simply supported in the $x$-direction along AD and $\mathrm{BC}$, while it is clamped in the $y$-direction along $\mathrm{AB}$ and DC. The three moments $M_{x}, M_{y}$, and $M_{x y}$ and the single transverse displacement $w$ are its response variables. The CBMF formulation for the plate flexure problem is obtained from the stationary condition of the IFM functional (ref. 3). The stationary condition yields three field equations in moment variables, containing one EE and two CCs:

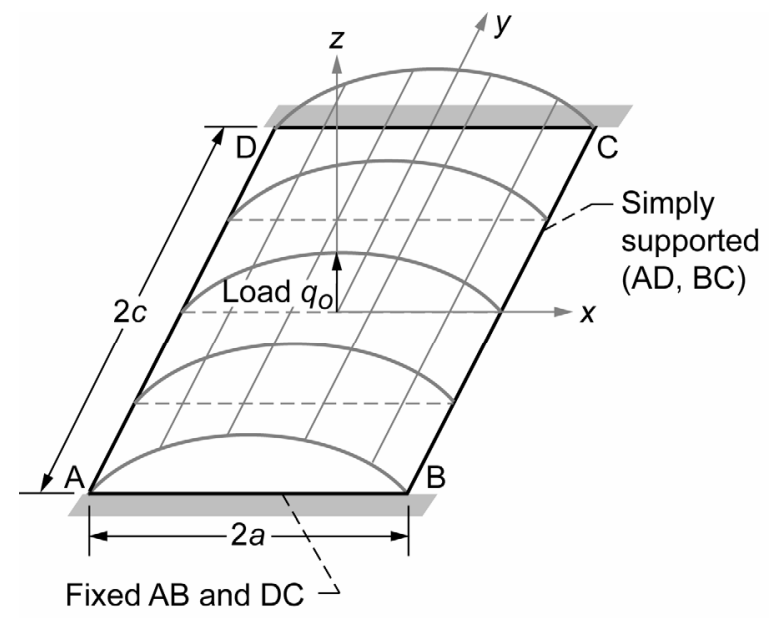

Figure 4.-Rectangular plate in flexure.

$$
\begin{gathered}
\mathrm{EE}: \frac{\partial^{2} M_{x}}{\partial x^{2}}+\frac{\partial^{2} M_{y}}{\partial y^{2}}+\frac{\partial^{2} M_{x y}}{\partial x \partial y}=q \\
\mathrm{CC}_{1}: \frac{\partial}{\partial x}\left(M_{y}-v M_{x}\right)-(1+v) \frac{\partial}{\partial y} M_{x y}=0 \\
\mathrm{CC}_{2}: \frac{\partial}{\partial y}\left(M_{x}-v M_{y}\right)-(1+v) \frac{\partial}{\partial x} M_{x y}=0
\end{gathered}
$$

The three field equations are reduced to two by eliminating $M_{x y}$ between the two CCs. The working equations of the $\mathrm{CBMF}$ and the boundary conditions are listed below:

Field equations of the plate flexure problem in CBMF

$$
\begin{gathered}
\nabla^{2}\left(M_{x}+M_{y}\right)=(1+v) q \\
\frac{\partial^{2}}{\partial x^{2}}\left(M_{y}-v M_{x}\right)-\frac{\partial^{2}}{\partial y^{2}}\left(M_{x}-v M_{y}\right)=0
\end{gathered}
$$

\section{Boundary condition of the plate flexure problem in CBMF}

Simply supported edges (AD and BC):

$$
\begin{aligned}
& M_{x}=0 \\
& M_{y}=0
\end{aligned}
$$

Clamped edges (AB and DC):

$$
\begin{gathered}
M_{x}-v M_{y}=0 \\
M_{x y}=0
\end{gathered}
$$

The field equation and boundary conditions of Navier's method, expressed in displacement $(w)$, are as follows

\section{Field equation in Navier's (or stiffness) method}

$$
\nabla^{4} w=\frac{q}{D}
$$

No explicit field CC because it is automatically satisfied in displacement. 


\section{Boundary condition in Navier's (or stiffness) method}

Simply supported edges (AD and $\mathrm{BC})$ :

$$
\begin{aligned}
& \frac{\partial^{2} w}{\partial x^{2}}+v \frac{\partial^{2} w}{\partial y^{2}}=0 \\
& w=0
\end{aligned}
$$

Clamped edges (AB and DC):

$$
\begin{aligned}
& \frac{\partial w}{\partial y}=0 \\
& w=0
\end{aligned}
$$

\section{Boundary condition in CBMF written in displacement}

Simply supported edges (AD and $\mathrm{BC})$ :

$$
\begin{aligned}
& \frac{\partial^{2} w}{\partial x^{2}}=0 \\
& \frac{\partial^{2} w}{\partial y^{2}}=0
\end{aligned}
$$

Clamped edges (AB and DC):

$$
\begin{aligned}
& \frac{\partial^{2} w}{\partial x^{2}}=0 \\
& \frac{\partial^{2} w}{\partial x \partial y}=0
\end{aligned}
$$

In the CBMF, the kinematics condition $w=0$ is not used to calculate the moments because it represents a rigid motion and does not induce moment or stress in the plate. During the back-calculation of displacement from moments, the CBMF method uses the rigid body displacement $(w=0)$ along the boundaries $\mathrm{AB}, \mathrm{BC}, \mathrm{CD}$, and $\mathrm{DA}$. The slope condition is not at all used in the CBMF. The field equations in the CBMF and the Navier's method are equivalent to a fourth-order equation. Each method has two boundary conditions at each edge. The boundary conditions are consistent with the order of the field equations for both methods. In other words, the new boundary compatibility conditions do not create an infeasibility issue.

2.2.1 Solution strategy.-In the CBMF the second-order differential equation (29a) is solved first to obtain the sum of moments $M_{x}+M_{y}$. Then equation (29b) is uncoupled and solved independently. The CBMF requires the solution of two second-order uncoupled differential equations. It is simpler than the stiffness method, where a fourth-order differential equation must be solved. The CBMF solution is obtained using separation of variables.

$$
\begin{aligned}
M_{x} & =\overline{M_{x}}(y) \cos \alpha x \\
M_{y} & =\overline{M_{y}}(y) \cos \alpha x \\
M_{x y} & =\overline{M_{x y}}(y) \sin \alpha x
\end{aligned}
$$

The CBMF moment $\left(M_{x}, M_{y}\right.$, and $\left.M_{x y}\right)$ solution follows:

$$
\begin{gathered}
M_{x}=\cos \alpha x\left[C_{3}\left(e^{\alpha y}+e^{-\alpha y}\right)\right. \\
\left.-\alpha \frac{(1-v)}{2(1+v)} C_{1} y\left(e^{\alpha y}-e^{-\alpha y}\right)-\frac{q_{0}}{\alpha^{2}}\right] \\
M_{y}=\cos \alpha x\left[\left(C_{1}-C_{3}\right)\left(e^{\alpha y}+e^{-\alpha y}\right)\right. \\
\left.+\alpha \frac{(1-v)}{2(1+v)} C_{1} y\left(e^{\alpha y}-e^{-\alpha y}\right)-v \frac{q_{0}}{\alpha^{2}}\right] \\
M_{x y}=\frac{\sin \alpha x}{2}\left[\left(2 C_{3}-C_{1}\right)\left(e^{\alpha y}-e^{-\alpha y}\right)\right. \\
\left.-\alpha \frac{(1-v)}{(1+v)} C_{1} y\left(e^{\alpha y}+e^{-\alpha y}\right)\right]
\end{gathered}
$$

Displacement $w$ is calculated by integrating a moment component. The integration constants are evaluated from the rigid-body boundary condition $w=0$. The displacement function follows:

$$
\begin{gathered}
w=\frac{-\cos \alpha x}{\alpha^{2} D\left(1-v^{2}\right)}\left[C_{3}(1+v)\left(e^{\alpha y}+e^{-\alpha y}\right)\right. \\
-v C_{1}\left(e^{\alpha y}+e^{-\alpha y}\right) \\
-\alpha \frac{(1-v)}{2} C_{1} y\left(e^{\alpha y}+e^{-\alpha y}\right) \\
\left.-\frac{q_{0}}{\alpha^{2}}\left(1-v^{2}\right)\right]
\end{gathered}
$$


The constants $C_{1}$ and $C_{3}$ are given by

$$
\begin{gathered}
C_{1}=\left(2 \frac{q_{0}}{\alpha^{2}}\right)(1+v) \frac{e^{\alpha c}-e^{-\alpha c}}{e^{2 \alpha c}+4 \alpha c-e^{-2 \alpha c}} \\
C_{3}=\frac{q_{0}}{\alpha^{2}} \frac{(1+v)\left(e^{\alpha c}-e^{-\alpha c}\right)+\alpha(1-v) c\left(e^{\alpha c}+e^{-\alpha c}\right)}{e^{2 \alpha c}+4 \alpha c-e^{-2 \alpha c}}
\end{gathered}
$$

Identical solutions are obtained for displacement and moments by Navier's method.

2.2.2 Boundary conditions.-The moment boundary conditions in the CBMF are expressed in terms of curvatures using the moment curvature relations to obtain equations (35) and (36). The CBMF boundary conditions, when expressed in displacement, do not identically map into the conditions of the displacement method (see eqs. (33) to (36)). In the CBMF, the boundary conditions are expressed in moments or curvatures, but in the displacement method these are written in moment, displacement, and slope. Consider for example, the simply supported boundary conditions along $\mathrm{AD}$ and $\mathrm{BC}$. The $\mathrm{CBMF}$ method imposes zero-moment conditions $M_{x}=M_{y}=0$ along the boundary. When expressed in displacement these become zero conditions in curvatures $\left(\frac{\partial^{2} w}{\partial x^{2}}=\frac{\partial^{2} w}{\partial y^{2}}=0\right)$. The boundary conditions in the CBMF and displacement methods appear to be different. The same logic applies to the clamped boundary. In the CBMF, conditions are expressed in curvatures $\left(\frac{\partial^{2} w}{\partial x^{2}}=\frac{\partial^{2} w}{\partial x \partial y}=0\right)$, while they are in terms of displacement and slope in Navier's method $\left(w=\frac{\partial w}{\partial y}=0\right)$. However, the response $\left(M_{x}, M_{y}, M_{x y}, w\right)$ is the same by either formulation because the boundary conditions of the two methods can be shown to be equivalent. This is explained next.

For simply supported boundaries along $\mathrm{AD}$ and $\mathrm{BC}(x=$ $-a$ and $x=a$, and $-c \leq y \leq c$ ), the displacement can be differentiated with respect to the $y$-coordinate without any consequence because the displacement is uniform across this boundary.

$$
w=0 \Rightarrow \frac{\partial w}{\partial y}=\frac{\partial^{2} w}{\partial y^{2}}=0
$$

and

$$
\left(\frac{\partial^{2} w}{\partial x^{2}}+v \frac{\partial^{2} w}{\partial y^{2}}=0\right)
$$

Thus,

$$
\frac{\partial^{2} w}{\partial x^{2}}=\frac{\partial^{2} w}{\partial y^{2}} \Rightarrow M_{x}=M_{y}=0
$$

For clamped boundaries along $\mathrm{AB}$ and DC $(y=-c$ and $y=$ $c$, and $-a \leq x \leq a$ ), we can differentiate the slope and displacement with respect to the $x$-coordinate.

$$
\frac{\partial w}{\partial y} \Rightarrow \frac{\partial^{2} w}{\partial x \partial y}=0
$$

and

$$
(w=0) \Rightarrow \frac{\partial w}{\partial x}=\frac{\partial^{2} w}{\partial x^{2}}=0
$$

Thus,

$$
M_{x y}=0
$$

and

$$
M_{x}-v M_{y}=0
$$

The boundary conditions become equivalent in Navier's and the CBMF methods. Thus both methods produce an identical response. The equivalence concept may become invalid if the rigid (or uniformity) condition (used in eqs. (40) and (41)) is not allowed, such as along a flexible finite element interface (ref. 17). All boundary conditions, including the continuity conditions, should be examined for equivalence between the CBMF and displacement method. A comprehensive comparison is not included in this paper because it requires substantial discussion.

\subsection{Summary on Elasticity}

In the theory of linear elasticity, Navier's method solved all three types of boundary value problems in elasticity, whereas the classical Beltrami-Michell formulation could not solve the second and third boundary value problems. In other words, the classical theory of elasticity was incomplete. Love's statement below was applicable only to the stress boundary value problem (ref. 14): 
"It is possible by taking account of these [compatibility] relations to obtain a complete system of equations [Beltrami-Michell Formulation] which must be satisfied by stress components, and thus the way is open for a direct determination of stress without the intermediate steps of forming and solving differential equations to determine the components of displacements."

A telltale sign pointing to the incompleteness was ignored. The BCC has alleviated the limitation of the classical Beltrami-Michell formulation. The completed method, CBMF, can solve all three classes of boundary value problems in elasticity. However, we might have exposed a new issue: for example, for a plate flexure problem, the boundary condition should be imposed on moments in the CBMF, which translates to curvatures in displacement method instead of the popular slope condition. Resolution of the issue requires further research.

\subsection{Structures}

The traditional compatibility condition in structures was formulated through a concept of redundant force. The CC was improvised by cutting a redundant member and subsequently closing the gap. The process automated by Denke (ref. 18) was referred to as "structure cutter." The fictitious "cut" and "close" gap technique is not even parallel to the then available strain formulation in elasticity. The force method based on the concept of redundant forces has disappeared for all practical purpose because it was clumsy and could not be extended to dynamic and buckling analyses (ref. 19). We have extended the strain formulation to structures (ref. 20). The CCs thus obtained are added to the equilibrium equation. This method with force as the primary variable is referred to as the "integrated force method" (IFM) (ref. 5). The IFM bestows simultaneous emphasis on the EE and the CC. Conceptually, the CBMF and IFM are parallel formulations in elasticity and in structures, respectively. To utilize the vast resources that have been developed for the regular stiffness method, like equation solver as well as preand postprocessors, a dual method to the primal IFM has been formulated. The dual integrated force method (IFMD) (ref. 11) with displacement as the primary unknown is obtained by transforming the equations of the IFM. Governing equations of the IFMD are symmetrical and resemble the stiffness equation in size and form. Both the IFM and IFMD produce an identical response. The IFMD can utilize the equation solvers of the popular stiffness method. In fact, a stiffness method code can be converted into IFMD software with minimal programming effort.

The formulation of the CC and IFM are introduced here through a simple example. Then the equations of the IFM and IFMD are given. Fidelity of the IFM and IFMD solutions is discussed.

\subsection{Illustrative Example}

The basic steps of the IFM that included the CC are introduced, considering the example of the three-bar truss shown in figure 5. The truss is made of steel with Young's modulus $E=30000 \mathrm{ksi}$, bar areas $A_{1}=A_{2}=1.0$ and $A_{3}=2.0 \mathrm{in}^{2}$, and load $P_{x}=50$ and $P_{y}=100 \mathrm{kip}$. The $n=3$ bar forces $F_{1}, F_{2}$, and $F_{3}$ and the $m=2$ displacements $X_{1}$ and $X_{2}$ are its five response variables. The structure has $m=2$ equilibrium equations, which are written at the free node 1 :

$$
\left[\begin{array}{ccc}
\frac{1}{\sqrt{2}} & 0 & \frac{-1}{\sqrt{2}} \\
\frac{-1}{\sqrt{2}} & -1 & \frac{-1}{\sqrt{2}}
\end{array}\right]\left\{\begin{array}{c}
F_{1} \\
F_{2} \\
F_{3}
\end{array}\right\}=\left\{\begin{array}{c}
P_{x} \\
P_{y}
\end{array}\right\}=\left\{\begin{array}{c}
50 \\
100
\end{array}\right\}
$$

$$
[B]\{F\}=\{P\}
$$

Here, $[B]$ is the rectangular equilibrium matrix of dimension $m \times n,\{F\}$ is the $n$-component force vector, and $\{P\}$ is the $m$-component load vector. Three forces cannot be determined from the two EE. One CC is required. This is obtained as an extension of the strain formulation in elasticity to structures (ref. 20). The CC is generated in three steps. First, the deformation-displacement $\{\beta\}-\{X\}$ relation (DDR) is obtained as $\{\beta\}=[B]^{T}\{X\}$. Then displacements are eliminated from the DDR to obtain the $\mathrm{CC}$ in terms of deformations. Finally, the deformations are eliminated in favor of bar forces using the flexibility relationship to obtain the $\mathrm{CC}$ in forces.

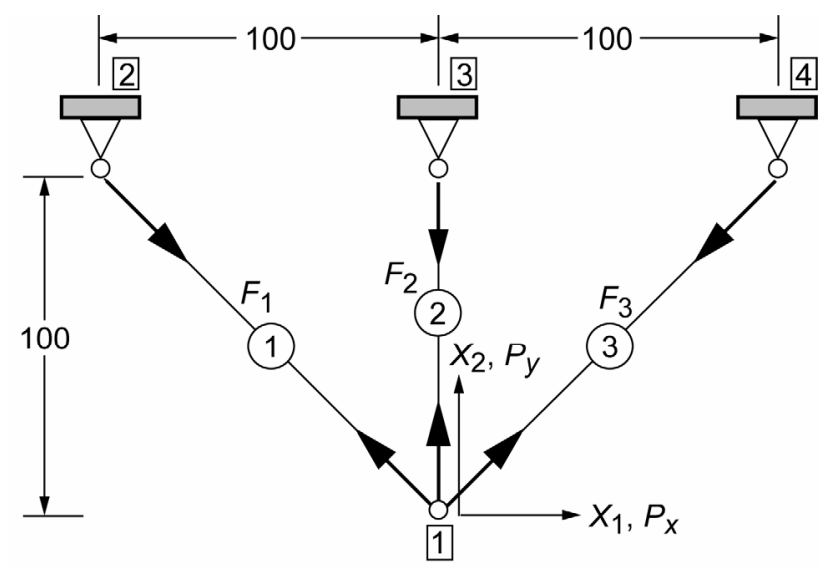

Figure 5.-Three-bar truss with loads $P$, bar forces $F$, and displacements $X_{1}$ and $X_{2}$. Dimensions are in inches. 
The DDRs $\{\beta\}=[\mathrm{B}] \mathrm{T}\{\mathrm{X}\}$ that are analogous to the strain displacement relation in elasticity follow:

$$
\left\{\begin{array}{l}
\beta_{1} \\
\beta_{2} \\
\beta_{3}
\end{array}\right\}=\left[\begin{array}{cc}
\frac{1}{\sqrt{2}} & -\frac{1}{\sqrt{2}} \\
0 & -1 \\
-\frac{1}{\sqrt{2}} & -\frac{1}{\sqrt{2}}
\end{array}\right]\left\{\begin{array}{l}
X_{1} \\
X_{2}
\end{array}\right\}
$$

Here, $\beta_{1}, \beta_{2}$, and $\beta_{3}$ are the bar deformations, and $X_{1}$ and $X_{2}$ are nodal displacements. The $\mathrm{CC}$ is obtained by eliminating the two displacements from the three strain displacement relations:

$$
\begin{gathered}
{\left[\begin{array}{lll}
1 & -\sqrt{2} & 1
\end{array}\right]\left\{\begin{array}{l}
\beta_{1} \\
\beta_{2} \\
\beta_{3}
\end{array}\right\}=\{0\}} \\
{[C]\{\beta\}=\{\delta R\}}
\end{gathered}
$$

Here, $[C]$ is the $r \times n$ (where $r=n-m$ ) compatibility matrix, and $\{\delta R\}$ is the $r$-component initial deformation vector for the problem; for a mechanical load, $\{\delta R\}=0$. The $\mathrm{CC}([C]\{\beta\}=0)$ is analogous to the strain formulation in elasticity.

The deformations are related to the forces $\{\beta\}=[G]\{F\}$ through the flexibility matrix $[G]$ of dimension $n \times n$ :

$$
\left\{\begin{array}{l}
\beta_{1} \\
\beta_{2} \\
\beta_{3}
\end{array}\right\}=\left[\begin{array}{ccc}
\left(\frac{\ell}{A E}\right)_{1} & 0 & 0 \\
0 & \left(\frac{\ell}{A E}\right)_{2} & 0 \\
0 & 0 & \left(\frac{\ell}{A E}\right)_{3}
\end{array}\right]\left\{\begin{array}{l}
F_{1} \\
F_{2} \\
F_{3}
\end{array}\right\}
$$

The $\mathrm{CC}$ is expressed in forces by eliminating deformation between equations (42d) and (42f):

$$
\left(\frac{100 \sqrt{2}}{E}\right)\left[\begin{array}{lll}
1 & -1 & \frac{1}{2}
\end{array}\right]\left\{\begin{array}{l}
F_{1} \\
F_{2} \\
F_{3}
\end{array}\right\}=0
$$

or

$$
[C][G]\{F\}=\{\delta R\}=\{0\}
$$

The EE $([B]\{F\}=\{P\})$ and the CC $([C][G]\{F\}=\{\delta R\}=$ $\{0\})$ are added to obtain the governing equation of the IFM as $[S]\{F\}=\{P\}$ :

$$
\left[\begin{array}{ccc}
\frac{1}{\sqrt{2}} & 0 & \frac{-1}{\sqrt{2}} \\
\frac{-1}{\sqrt{2}} & -1 & \frac{-1}{\sqrt{2}} \\
1 & -1 & \frac{1}{2}
\end{array}\right]\left\{\begin{array}{l}
F_{1} \\
F_{2} \\
F_{3}
\end{array}\right\}=\left\{\begin{array}{c}
50 \\
100 \\
0
\end{array}\right\}
$$

Solution to equation (42i) yields the forces as

$$
\left\{\begin{array}{l}
F_{1} \\
F_{2} \\
F_{3}
\end{array}\right\}=\left\{\begin{array}{c}
5.025 \\
42.893 \\
75.736
\end{array}\right\}
$$

The displacements are back calculated from forces using a formula $\{X\}=[J][G]\{F\}$, where $[J]=m$ rows of $[S]^{-T}$.

$$
\left\{\begin{array}{l}
u \\
v
\end{array}\right\}=\left(\frac{100}{E}\right)\left\{\begin{array}{c}
F_{2} \\
2 F_{1}-F_{2}
\end{array}\right\}=\left\{\begin{array}{l}
0.143 \\
0.110
\end{array}\right\}_{\text {in. }}
$$

The generalization of the calculations shown for the threebar truss became the IFM. The IFM, which is the discrete analogue of the CBMF, simultaneously emphasized the EE and the CC. The IFM was not developed earlier because the $\mathrm{CC}$ was not available.

\subsection{Equations for the Integrated Force Method}

In the IFM of analysis, a finite element model, is characterized by two attributes: $n$ forces $\{F\}$ and $m$ displacements $\{X\}$. The IFM has one set of equations to calculate the internal forces and another set to back-calculate the displacements. The IFM equations for static and free-vibration (or frequency) analyses are as follows:

Static analysis:

$$
[S]\{F\}=\left\{P^{*}\right\}
$$

$$
\{X\}=[J]\left\{[G]\{F\}+\left\{\beta^{0}\right\}\right\}
$$

where $[S]=\left[\begin{array}{c}{[B]} \\ {[C][G]}\end{array}\right],[J]=m$ rows of $\left([S]^{-1}\right)^{T}$, and

$\left\{P^{*}\right\}=\left\{\begin{array}{c}\{P\} \\ \hdashline \delta R\}=-[C]\left\{\beta^{0}\right\}\end{array}\right\}$. 
Free-vibration analysis:

$$
\left([S]-\omega^{2}\left[\frac{[M][J][G]}{[0]}\right]\right)\{F\}=\{0\}
$$

Calculation of displacement mode shape in frequency analysis can use the displacement calculation formula given by equation (44) in static analysis. The symbols in the static and free-vibration analyses are listed as follows:

$\{F\} \quad n$ component internal force vector

$\{P\} \quad m$ component external load vector

$\{\delta R\} \quad(r=n-m)$ component effective initial deformation vector

$\left\{\beta^{0}\right\} \quad n$ component initial deformation vector

$\{\beta\} \quad n$ component deformation vector

$\{X\} \quad m$ component displacement vector

$[S] \quad n \times n$ IFM governing matrix

[B] $\quad m \times n$ equilibrium matrix

[C] $\quad r \times n$ compatibility matrix

$[G] \quad n \times n$ flexibility matrix

[J] $\quad m \times n$ displacement coefficient matrix

[M] $\quad m \times m$ mass matrix

$m \quad$ number of displacement unknowns

$n \quad$ number of force unknowns

$\omega \quad$ circular frequency

3.2.1 Dual Integrated Force Method.-Solution of a symmetrical set of equations is very well developed, especially because of its use in the finite element stiffness method. Furthermore, the Patran pre- and postprocessing software (MSC.Software Corporation) is a very useful analysis tool. The IFM can benefit from the solver and the software, provided the IFM equation can be cast in the format of the stiffness method. This has been achieved, and the method is referred to as the dual integrated force method (IFMD). The equations of the IFMD are obtained by mapping forces into displacement (ref. 11). Like the IFM, the dual method also has two sets of equations. The first set is used to calculate displacement, while the second set backcalculates forces. The primal and dual methods produce identical solutions for force, displacement, and frequency. The equations of the IFMD follow:

Static analysis:

$$
\begin{gathered}
{[D]\{X\}=\{P\}+\left\{P^{0}\right\}} \\
\{F\}=[G]^{-1}\left\{[B]^{T}\{X\}-\left\{\beta^{0}\right\}\right\}
\end{gathered}
$$

where $[D]=[B][G]^{-1}[B]^{T}$ and $\left\{P^{0}\right\}=[B][G]^{-1}\left\{\beta^{0}\right\}$
Frequency analysis:

$$
\left[[D]-\omega^{2}[M]\right]\{X\}=\{0\}
$$

Here, [D] is an $m \times m$ governing matrix of the IFMD, and $\left\{P^{0}\right\}$ is the initial load vector. Calculation of force mode shape in frequency analysis can use the force calculation formula in static IFM analysis given by equation (46b). The governing equation of the IFMD resembles that of the regular stiffness method, $[K]\{X\}=\left\{P^{\text {stiff }}\right\}$, where $[\mathrm{K}]$ is the $m \times m$ stiffness matrix, and $\left\{P^{\text {stiff }}\right\}$ is the $m$-component load vector of the stiffness method. Both $[K]$ and $[D]$ are symmetrical matrices with identical dimensions, but their coefficients are not equal $\left(D_{i j} \neq K_{i j}\right)$. Notice the flexibility matrix $[G]$, which is maintained in a pristine state in both the primal and the dual methods. This makes the calculation of design sensitivity straightforward via the IFM and IFMD.

3.2.2 Other methods. - The matrices of the IFM can be used to formulate the mixed method and the total formulation of structural mechanics given in table I. The reverse course cannot be followed. For example, the equations of the stiffness method cannot be transformed to obtain the IFM. However, the IFM equations were specialized to obtain the IFMD, which in essence is the displacement method. Likewise, the mixed method and the total formulation can be constructed from the IFM equations.

3.2.2.1 Mixed method: The mixed method treats force $\{F\}$ and displacement $\{X\}$ as the simultaneous unknowns. The governing equation of this method is obtained by concatenating the two IFM equations as

$$
\left[\frac{[S]}{[-[J][G]][I]}\right]\left\{\begin{array}{l}
\{F\} \\
\{X\}
\end{array}\right\}=\left\{\frac{\left\{P^{*}\right\}}{[J]\left\{\beta^{0}\right\}}\right\}
$$

where $[I]$ is the identity matrix.

3.2.2.2 Total formulation: The total formulation considers force $\{F\}$, displacement $\{X\}$, and deformation $\{\beta\}$ as the simultaneous unknowns. The governing equation of the total formulation is obtained by adding the flexibility formula $[G]\{F\}=\{\beta\}$ to the equations of the mixed method.

$$
\left[\begin{array}{ccc}
{[S]} & 0 & 0 \\
-[J][G] & {[I]} & 0 \\
-[G] & 0 & {[I]}
\end{array}\right]\left\{\begin{array}{c}
\{F\} \\
\{X\} \\
\{\beta\}
\end{array}\right\}=\left\{\begin{array}{c}
\left\{P^{*}\right\} \\
{[J]\left\{\beta^{0}\right\}} \\
\{0\}
\end{array}\right\}
$$

The discussion to follow emphasizes only the force method (IFM) and the dual method (IFMD). The popular stiffness method is used to compare results. 
3.2.3 Solution fidelity.-The stiffness method is a popular research topic throughout the world. Commercial code developers are continuously improving the performance of this method through techniques like reduced integration, the use of a bubble function, and others. Also, the IFMD concept might have been incorporated (ref. 21) into the stiffness method. It is difficult to numerically compare with such improved techniques in the commercial software because of too many attributes, some of which may even be classified. For a meaningful comparison in a controlled environment we have developed the IFM/Analyzers software (ref. 22). This finite element code incorporates the IFM and IFMD as well as the standard stiffness method. The code has a total of 44 different types of elements for each method. The generation of each element involves similar energy expressions as well as the same interpolation polynomials and numerical integration technique. The IFM/Analyzers element library includes beam, membrane, plate, and solid elements of different shapes. Some elements have midside nodes. IFM/Analyzers is a Fortran 77 code, written for both sequential and parallel calculations. It performs linear analysis for mechanical and thermal loads and initial displacement as well as frequency analysis. The analyzer uses the NASA GPS sparse solver (ref. 23) and Harwell routines (ref. 24). Solutions have been obtained for finite element models up to one-half million unknowns. The IFM/Analyzers code has also been reduced to obtain a modest code with all three methods. The small code named "IFM-UE" can be used for undergraduate education in engineering. The code accompanies the textbook, reference 5 . The solution capacity of this code is about 5000 equations. It has five different types of elements that can model skeletal frames and membrane structures. The IFM, IFMD, and stiffness method have been compared in a controlled environment for a set of test problems (refs. 11 and 22). Solution is given for a few typical examples to illustrate the robustness of the IFM/Analyzers code.

3.2.3.1 A cantilever plate with settling of support: A cantilever plate $\mathrm{ABCD}$ is supported along $\mathrm{AD}$ with prescribed displacements $\bar{u}$ and $\bar{v}$ along this edge $\left(x=0,-\frac{h}{2} \leq y \leq \frac{h}{2}\right)$, as shown in figure 6(a). The prescribed displacement functions are

$$
\begin{gathered}
\bar{u}=\frac{4(2+v)}{3 E h^{2}} y\left(\frac{h^{2}}{4}-y^{2}\right) \\
\bar{v}=\frac{4 v L}{E h^{2}} y^{2}
\end{gathered}
$$

Mechanical load $\bar{\tau}$ applied along the free edge BC is

$$
\bar{\tau}=1-4 \frac{y^{2}}{h^{2}}
$$
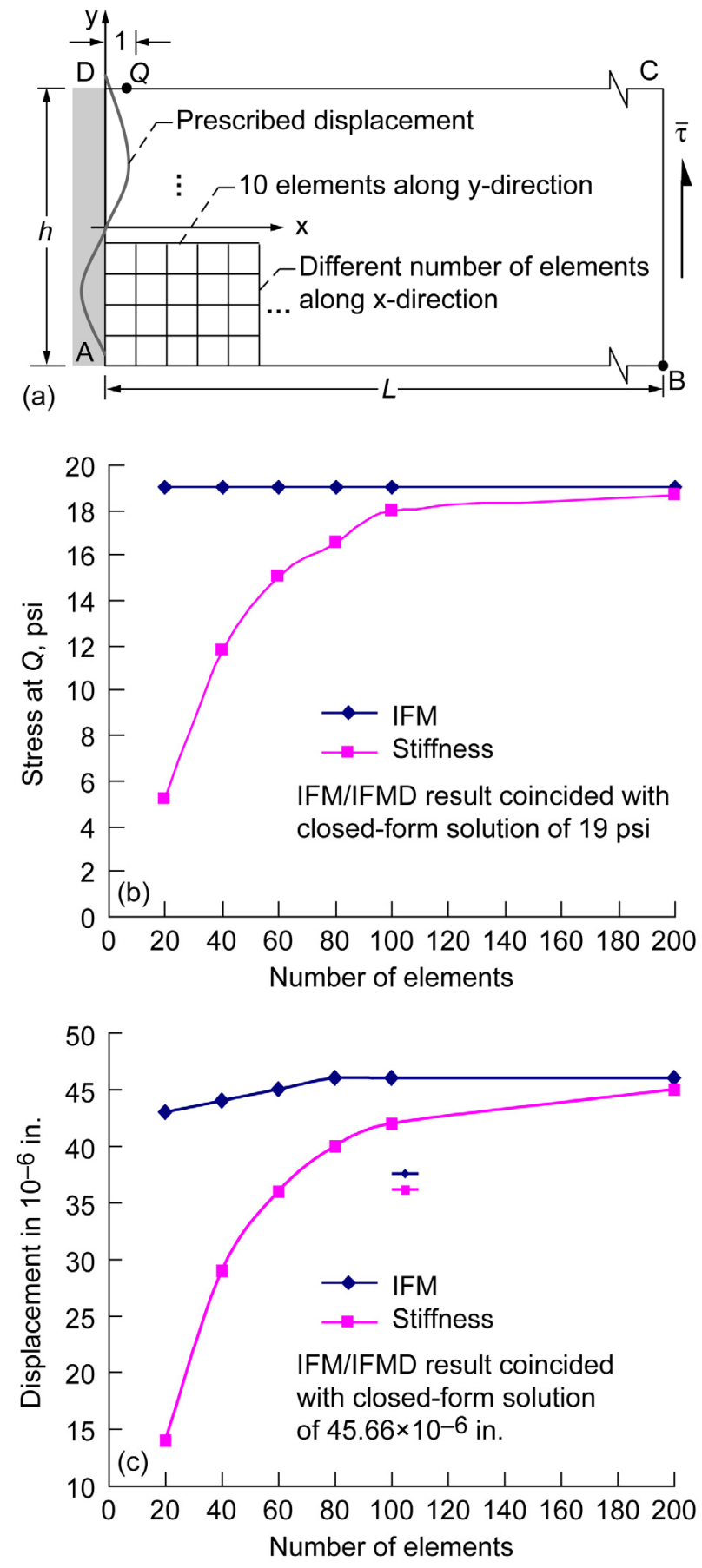

Figure 6.-Stress and displacement solutions for cantilever plate. (a) Cantilever plate. (b) Convergence of stress at location $Q$. (c) Convergence of displacement at location $B$.

The example has been used to provide a benchmark solution for finite element analysis. The analytical response, consisting of displacements $u$ and $v$ is as follows (ref. 25):

$$
u=\frac{4}{E h^{2}}\left[y\left(x^{2}-2 L x\right)+\frac{2+v}{3} y\left(\frac{h^{2}}{4}-y^{2}\right)\right]
$$




$$
v=\frac{4}{E h^{2}}\left[L x^{2}-\frac{x^{3}}{3}-v y^{2}(x-L)+\frac{4+5 v}{12} h^{2} x\right]
$$

A finite element method solution was obtained using the IFM and stiffness method. A simple quadrilateral membrane element was used. The stiffness element had 8 displacement degrees of freedom. The IFM considered five force unknowns (ref. 5) with the displacement function that is identical to the stiffness method. The parameters in the problem were length $L=20 \mathrm{in}$., height $h=1.0 \mathrm{in}$., thickness $t$ $=0.125$ in., Young's modulus $E=30000 \mathrm{ksi}$, and Poisson's ratio $v=0.3$. Normal stress $\sigma_{x}^{\mathrm{Q}}$ is calculated at the top fiber location Q with coordinates $x=1.0$ in. and $y=0.5$ in. as shown in figure 6(a). Transverse displacement $v^{\mathrm{B}}$ is determined at location B with coordinates $x=20$ in. and $y=-$ $0.5 \mathrm{in}$. The analytical solution for stress and displacement are as follows:

$$
\begin{aligned}
\sigma_{x}^{\mathrm{Q}} & =19 \mathrm{psi} \\
v^{\mathrm{B}} & =45.66 \times 10^{-6} \text { in. }
\end{aligned}
$$

The finite element solutions are given in table II. Ten finite elements were used along the $y$-coordinate direction to accommodate the variation of load and prescribed displacement given in equations (50) to (52). Along the $x$-coordinate direction, the number of elements was progressively changed from 2 to 20 . A 70 -element model for example, had 7 and 10 elements along the length and depth, respectively.

TABLE II.-STRESS AND DISPLACEMENT SOLUTIONS FOR CANTILEVER PLATE SHOWN IN FIGURE 6(a)

\begin{tabular}{|c|r|c|c|c|}
\hline & \multicolumn{2}{|c|}{$\begin{array}{c}\text { Stress } \sigma_{x}^{\mathrm{Q}} \\
\text { psi }\end{array}$} & \multicolumn{2}{c|}{$\begin{array}{c}\text { at }{ }^{\mathrm{a}, \mathrm{b}} \\
10^{-6} \text { in. }\end{array}$} \\
\hline Model & IFM/IFMD & Stiffness & IFM/IFMD & Stiffness \\
\hline $2 \times 10$ & $18.99(100)$ & $5.18(27.0)$ & $43(94.0)$ & $14(30.2)$ \\
\hline $4 \times 10$ & $19(100)$ & $11.80(62.0)$ & $45(98.5)$ & $29(62.9)$ \\
\hline $6 \times 10$ & $19(100)$ & $15.04(79.0)$ & $45(99.4)$ & $36(79.1)$ \\
\hline $8 \times 10$ & $19(100)$ & $16.60(87.0)$ & $46(99.7)$ & $40(87.0)$ \\
\hline $10 \times 10$ & $19(100)$ & $17.97(94.6)$ & $46(99.8)$ & $42(91.2)$ \\
\hline $20 \times 10$ & $19(100)$ & $18.71(98.5)$ & $46(100.0)$ & $45(97.6)$ \\
\hline
\end{tabular}

${ }^{a}$ Numbers in parenthesis represent percentages of analytical value.

${ }^{\mathrm{b}}$ Position on plate in figure $6(\mathrm{a}), x=1$ and $y=-0.5 \mathrm{in}$.

${ }^{\mathrm{c}}$ Position on plate in figure $6(\mathrm{a}), x=20$ and $y=-0.5 \mathrm{in}$.

The primal (IFM) and dual (IFMD) methods yielded identical solutions. For the 20 -element $(2 \times 10)$ model, the IFM/IFMD code yielded a stress value of $\sigma_{x}^{\mathrm{Q}}=19 \mathrm{psi}$, which was identical to the analytical solution. The stiffness method, for the $2 \times 10$ model, yielded a stress of $\sigma_{x}^{\mathrm{Q}}=$ $5.2 \mathrm{psi}$, which was only 27 percent of the analytical solution. For other, dense models the IFM/IFMD result continued to coincide with the analytical solution as shown in figure $6(\mathrm{~b})$, while the stiffness method converged to a stress value that retained a 1.5 percent residual error, even for a large model with 200 elements. For the 20-element model, the IFM/IFMD code yielded a displacement value of $43 \times 10^{-6} \mathrm{in}$. compared with the analytical solution of $46 \times 10^{-6}$ in. The stiffness method, for that model, yielded a displacement value of $v^{B}=14 \times 10^{-6} \mathrm{in}$., which was only 30 percent of the analytical solution. For other, dense models the IFM/IFMD result coincided with the analytical solution as shown in figure 6(c), while the stiffness method converged to a displacement value that retained a 6 percent residual error using the biggest model with 200 elements.

3.2.3.2 A plate flexure problem: The rectangular plate under a concentrated load $P=1 \mathrm{kip}$, shown in figure 7(a), was a finite element test problem. It was made of steel with Young's modulus $E=30000 \mathrm{ksi}$, Poisson's ratio $\mathrm{v}=0.3$, thickness $t=0.25$ in., length $a=24$ in., and width $b=12$ in. It was clamped along all four edges. The analytical solution for the transverse displacement under the load was $w_{P}=$ $2.42 \times 10^{-3}$ in., and the moment at $x=0, y=b / 2$ was $M_{B}=167 \mathrm{lbf}$-in./in. A four-node quadrilateral element (ref. 9) was used to solve the problem. Each node has 3 degrees of freedom consisting of a displacement and two rotations $\left(w_{i}, \theta_{x i}, \theta_{y i}\right)$. A standard cubic polynomial with 12 unknowns was used to approximate the displacement function $w(x, y)$. For the IFM/IFMD code, the force interpolation contained nine unknowns. Linear variation was considered for the normal moments $M_{x}$ and $M_{y}$ with four unknowns each, while the twisting moment was uniform across the element. The displacement and moment solutions for the plate are graphed in figures 7(b) and (c), respectively. Convergence was achieved for IFM/IFMD in displacement and moment for a model with eight elements. The stiffness method from two commercial codes were used. These methods required 64 or more elements for convergence with some residual errors.

3.2.3.3 Frequency analysis of a turboprop blade: The turboprop blade, shown in figure 8(a), was made of steel with a weight density of $\rho=0.289 \mathrm{lbf} / \mathrm{in}^{3}{ }^{3}$ It had a spatial distribution of mass with a uniform thickness of $0.1 \mathrm{in}$. It was about $10 \mathrm{in}$. long and $4 \mathrm{in}$. wide at the midspan location. An eight-node brick element was used to calculate its frequency. The element had 24 degrees of freedom that correspond to three displacements at a node. The IFM element (ref. 22) used a total of 18 force unknowns: 4 for each normal stress and 2 for each shear stress. The brick element of a commercial code was comparable to the stiffness method of the IFM/Analyzers software. The frequency analysis results obtained by IFM/IFMD are given in table III. The convergence of frequency versus model size is shown in figure 8(b). A logarithmic scale was used along the $\mathrm{x}$-axis to accommodate the size of the large finite element models.

Solution from a commercial code is also included because the research-level IFM/Analyzers software cannot solve an 

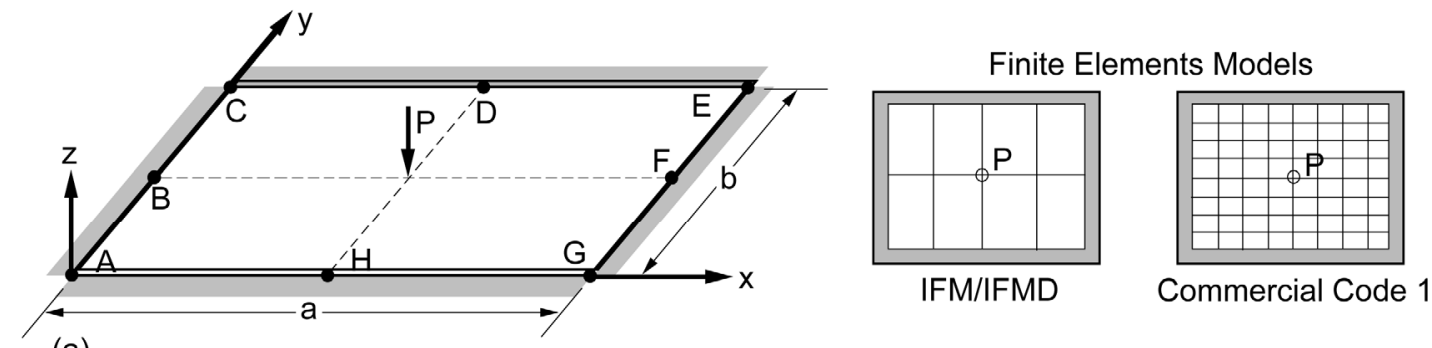

(a)
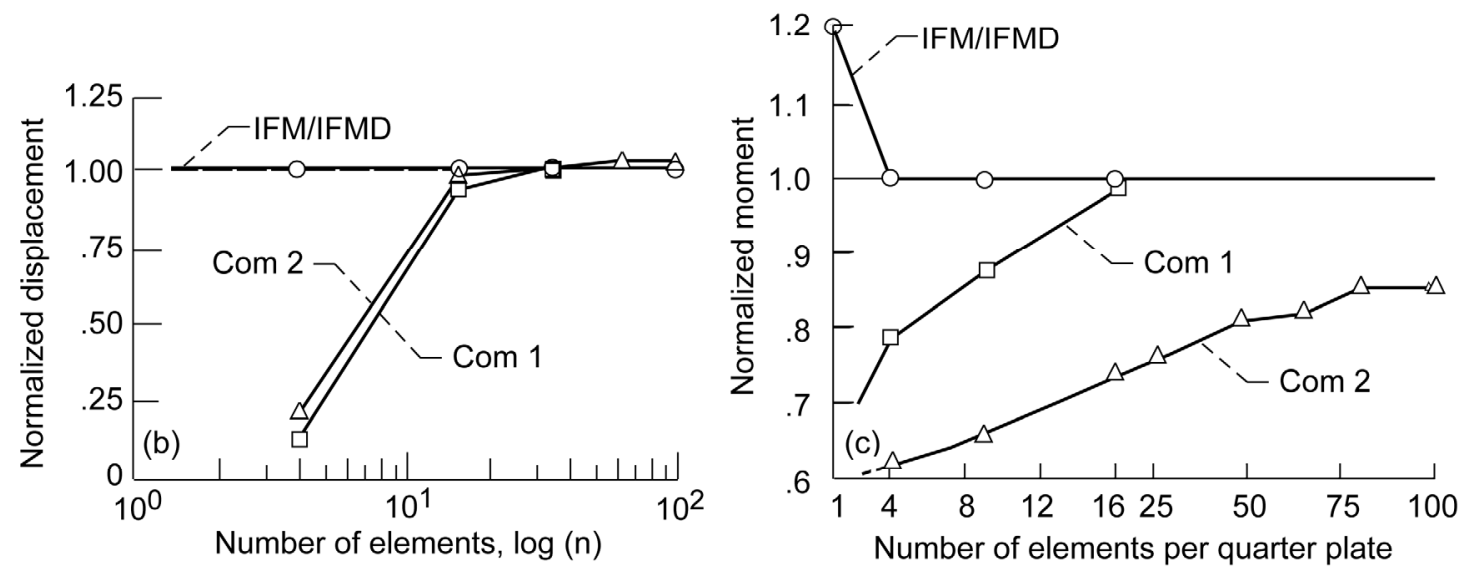

Figure 7.- Stress and displacement solutions for clamped plate. (a) Finite element model for clamped plate. (b) Convergence of displacement. (c) Convergence of moment.

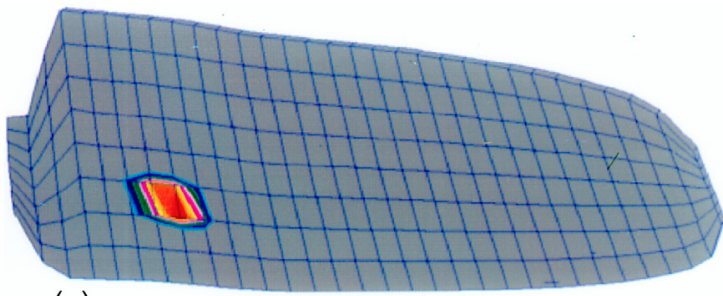

(a)

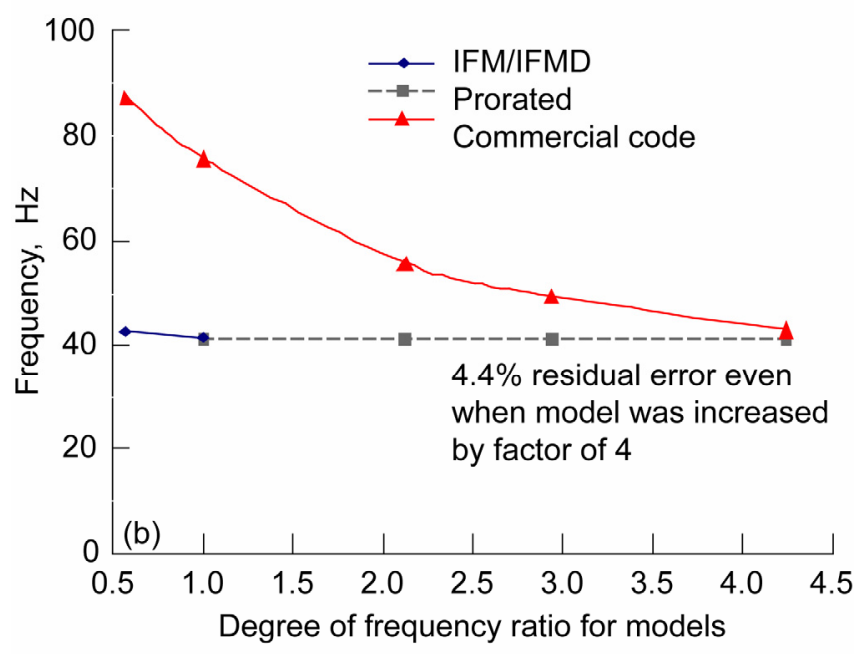

Figure 8.-Frequency analysis of turboprop blade. (a) Finite element model. (b) Convergence of frequency.
TABLE III.-FUNDAMENTAL FREQUENCIES BY DIFFERENT METHODS

\begin{tabular}{|l|c|c|c|}
\hline \multirow{2}{*}{$\begin{array}{l}\text { Model: } \\
\text { (number of elements, } \\
\text { degrees of freedom) }\end{array}$} & \multicolumn{3}{|c|}{$\begin{array}{c}\text { Frequency, } \\
\text { Hz }\end{array}$} \\
\cline { 2 - 4 } & IFM/IFMD & $\begin{array}{c}\text { Stiffness } \\
\text { method }\end{array}$ & $\begin{array}{c}\text { Commercial } \\
\text { code }\end{array}$ \\
\hline 1: $(132,924)$ & 42.5 & 87.5 & 87.5 \\
\hline $2:(240,1620)$ & 41.3 & 75.8 & 75.8 \\
\hline 3: $(528,3432)$ & ----- & ----- & 55.8 \\
\hline 4: $(720,4752)$ & ----- & ---- & 49.5 \\
\hline
\end{tabular}

eigenvalue problem with 3452 degrees of freedom in the model. The brick element of the commercial code is comparable to that of the stiffness method of the IFM/Analyzers software. The IFM/IFMD code produced a satisfactory frequency result for the first model with 924 degrees of freedom. (The next model with 1620 degrees of freedom yielded only a marginal 3-percent improvement.) The stiffness method and the commercial code produced much higher values for the frequency. The commercial code produced a frequency value of $87.5 \mathrm{~Hz}$ for the first model. For the last model with 6864 degrees of freedom the frequency was $43.1 \mathrm{~Hz}$. Frequency calculated by the stiffness method converged with a 4.4 percent residual error as shown in figure $8(\mathrm{~b})$.

The animation of the stress mode shape in IFM/IFMD (ref. 22) detected a stress concentration region in the same 
blade due to a small hole, which is qualitatively shown in figure 8(a). Typically, stress mode shape is not available in the stiffness formulation.

\subsection{Summary on Structures}

For the three examples given in this paper and from solutions of many other problems it was observed that the IFM/IFMD produced high-fidelity solutions with a modest finite element model. The stiffness method required a very large model; even then the solution accuracy could not be guaranteed. The monopolistic dominance of the stiffness method can only be justified because we can solve a very large number of equations with a computer. The IFM/IFMD methods produced accurate stress, displacement, and frequency results even for modest finite element models because of their simultaneous emphasis on the equilibrium equations and the compatibility conditions. The stiffness method lacked precision because this method neglected the compatibility conditions, especially along the numerous boundaries of a finite element model. Even though the stiffness method may be preferred at present because of the availability of well-written software, the development of a commercial code based on IFM/IFMD should be initiated.

\subsection{Design of Structures}

Traditionally the concept developed to design a determinate structure is also used to design an indeterminate structure. Consider for example a determinate truss. The concept of fully stressed design (FSD) works very well for a determinate truss. Typically, the FSD concept is extended to design an indeterminate truss. This becomes problematic (ref. 13) because an indeterminate truss cannot be fully stressed. Rules to design a determinate structure should be modified prior to their application to an indeterminate structure. The compatibility conditions are key ingredients that should be accounted for the modification because the CCs imposed implicit constraints in the design of an indeterminate structure. It turns out the $\mathrm{CC}$ has considerable influence on the fully stressed and fully utilized designs as well as in the optimization methods. The implicit constraints are intrinsic to the design of an indeterminate structure and are independent of the choice of an analysis method used in the design calculations. The nature of the constraints is easily studied via the IFM, however. The rules thus generated should be adopted even when the stiffness method analysis tool is used in design calculations.

Cilley (ref. 26), in 1900 discussed some aspects of design of an indeterminate truss. Reinschmidt et al. (ref. 27) attempted an analytical explanation through an illustrative example. Gallagher and Zienkiewicz (ref. 28) has also touched upon the issue. Some of our observations on the topic are reported in references 13,29, and 30. The central design issue is illustrated by considering truss examples.
Extension to indeterminate beams, framework, and other types of structures is straightforward, though it would require extensive algebraic manipulation. Both traditional design and optimization concepts are examined for an indeterminate truss, and the discussion is presented under three topics:

(1) Design for strength

(2) Design for stiffness

(3) Design optimization

\subsection{Design for Strength}

Design for strength attempts a full stress state for all members of the truss. Consider a truss with $n$ bars. For all bars in the truss a full stress condition is defined as

$$
\sigma_{i}=\sigma_{0}(i=1,2, \ldots, n)
$$

where $\sigma_{i}$ is stress in the $i^{\text {th }}$ bar and $\sigma_{0}$ is the strength. A stress ratio rule yields the area $A_{i}$ of the $i^{\text {th }}$ truss bar as a ratio of member force $F_{i}$ to strength: $A_{i}=F_{i} / \sigma_{0}$. The rule works well for a determinate truss but becomes problematic for an indeterminate truss.

4.1.1 Design of a determinate truss. - Consider a determinate truss with $n$ bars and the same number of bar areas. The bar areas can be calculated from the governing equation of the IFM $[S]\{F\}=\left\{P^{*}\right\}$ by expressing it in areas $A_{i}=F_{i} / \sigma_{0}$ to obtain $\left[S^{*}\right]\{A\}=\left\{P^{*}\right\}$. For a determinate structure the governing matrix is equal to the equilibrium matrix $([S]=[B])$, which is independent of bar areas. In other words, a single inversion without iteration yields the areas

$$
\{A\}=\operatorname{abs}\left\{\left[S^{*}\right]^{-1}\left\{P^{*}\right\}\right\}
$$

The formula in equation (56) can be extended to include buckling limitations.

Consider the two-bar determinate truss shown in figure 9(a). It is made of steel and is subjected to two load components, $P_{x}=10$ kip and $P_{y}=-20$ kip. The bar force is linked to the cross-sectional area $A$ through the yield stress as $F_{1}=\sigma_{0} A_{1}$ and $F_{2}=\sigma_{0} A_{2}$. The IFM governing equation degenerates into the equilibrium equations. The EEs are modified to obtain the design.

$$
\begin{aligned}
& {[B]\{F\}=\{P\} \Rightarrow[B]\{\sigma A\}=\{P\} \Rightarrow[B]\{A\}=\left(\frac{1}{\sigma_{0}}\right)\{P\}} \\
& \text { or }\{A\}=\operatorname{abs}\left\{\left(\frac{1}{\sigma_{0}}\right)[B]^{-1}\{P\}\right\}
\end{aligned}
$$



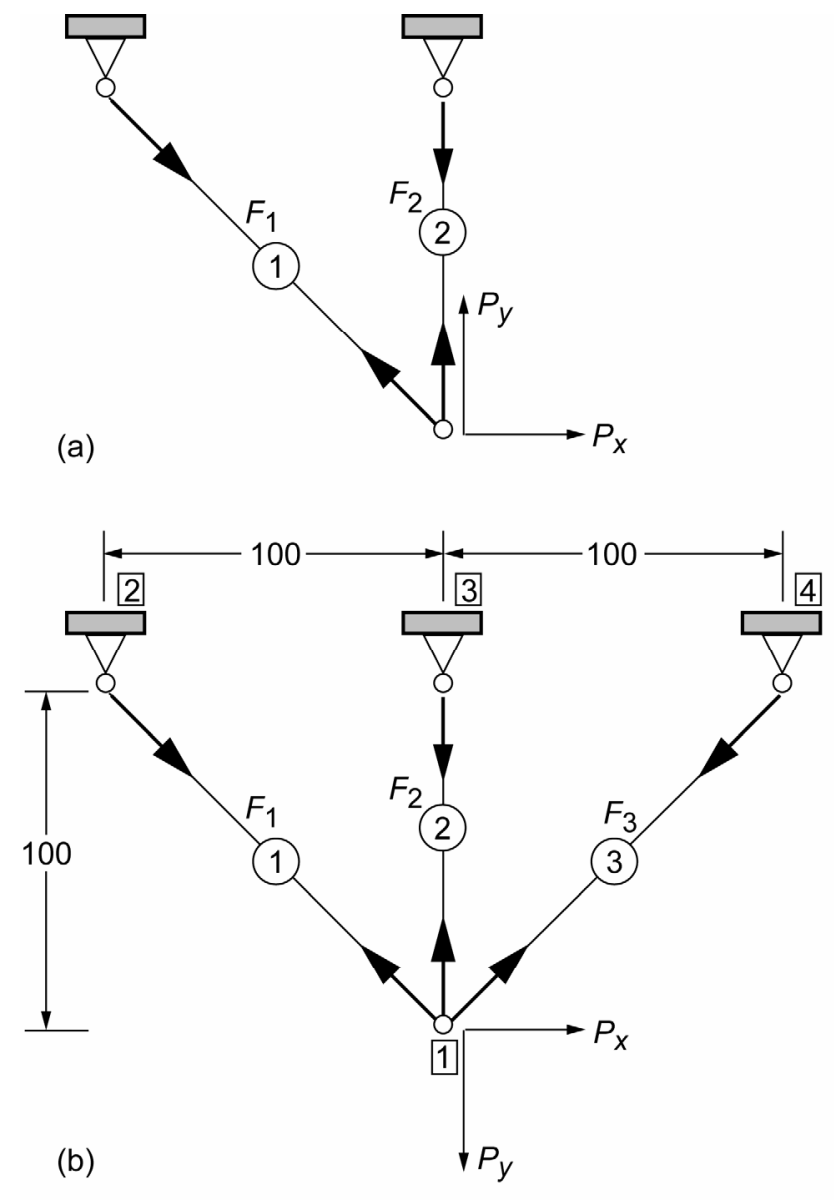

Figure 9.-Design of indeterminate truss. (a) Determinate.

(b) Indeterminate. Dimensions are in inches.

The bar areas are determined from equation (57) in a single step because the equilibrium matrix $[B]$ is independent of the design variables. The numerical values for the areas are as follows:

$$
\{A\}^{\text {two bar }}=\left\{\begin{array}{l}
0.71 \\
0.50
\end{array}\right\} \text { in. }^{2} \text { and weight }=43.4 \mathrm{lbf}
$$

4.1.2 Design of an indeterminate truss. - The truss is made indeterminate by adding a third bar as shown in figure 9(b). The following design equation is obtained when the determinate design concept is extended to the indeterminate truss:

$$
\begin{aligned}
& {[S]\{F\}=\left\{P^{*}\right\}} \\
& \text { or }\{A\}=\operatorname{abs}\left\{\left(\frac{1}{\sigma_{0}}\right)[S]^{-1}\left\{P^{*}\right\}\right\}
\end{aligned}
$$

Equation (58) has to be solved iteratively since the $[S]$ matrix is a function of bar areas because of the flexibility matrix $[G]$ in the compatibility condition $[C][G]\{F\}=\{0\}$.
Iterative solution of equation (58) yielded the following design:

$$
\{A\}^{\text {three bar }}=\left\{\begin{array}{l}
0.71 \\
0.50 \\
0.00
\end{array}\right\} \text { in. }^{2} \text { and weight }=43.4 \mathrm{lbf}
$$

An extension of the determinate design concept to an indeterminate structure returned with a zero area for the third bar. Furthermore, design and weight are identical for both determinate and indeterminate structures. This observation is not coincidental but is common to all indeterminate trusses. The implicit compatibility constraint distinguished between the designs of the determinate and indeterminate structures. This implicit compatibility constraint in deformation, force, and stress variables $\{\beta\},\{F\}$, and $\{\sigma\}$, respectively, is as follows:

$$
\begin{gathered}
\beta_{1}-\sqrt{2} \beta_{2}+\beta_{3}=0 \\
\left(\frac{F \ell}{A E}\right)_{1}-\sqrt{2}\left(\frac{F \ell}{A E}\right)_{2}+\left(\frac{F \ell}{A E}\right)_{3}=0 \\
\sigma_{1}-\sigma_{2}+\sigma_{3}=0
\end{gathered}
$$

The compatibility constraint is violated (ref. 13) when a fully stressed state $\left(\sigma_{1}=\sigma_{2}=\sigma_{3}=\sigma_{0}\right)$ is attempted: $\sigma_{1}-\sigma_{2}+$ $\sigma_{3} \Rightarrow 20-20+20 \neq 0$. Because of the violation, a bar area of zero is obtained for the third bar $\left(A_{3}=0\right)$ of the determinate truss. This observation can be extended for a general indeterminate truss with $n$ bars and $r$ redundant members. For such a truss $(n, m)$ there are $m=n-r$ equilibrium equations and $r=n-m$ compatibility conditions. A fully stressed condition cannot be achieved for an indeterminate truss $(n, m)$. An attempt to fully stress this truss would degenerate it to a determinate truss with areas of zero for $r$ number of bars: $A_{j}=0$ where $j=1, \ldots, r=n-m$.

4.1.3 Linking of design variables. - The situation associated with bar areas of zero in the design of an indeterminate truss can be alleviated by linking the design variables. The number of independent variables must be reduced to $m$ for an $n$-bar truss with $r$ redundant members. For the three-bar truss (see fig. 9(b)), we discuss two of several choices to link areas: link the areas of bar 1 and bar $3\left(A_{1}, A_{2}, A_{3}=A_{1}\right)$; likewise link $A_{2}$ and $A_{3}$. The designs obtained for the two cases are as follows:

$$
\{A\}^{A_{1} \text { and } A_{3}}=\left\{\begin{array}{l}
0.64 \\
0.66 \\
0.64
\end{array}\right\} \text { in. }^{2} \text { and weight }=71.5 \mathrm{lbf}
$$




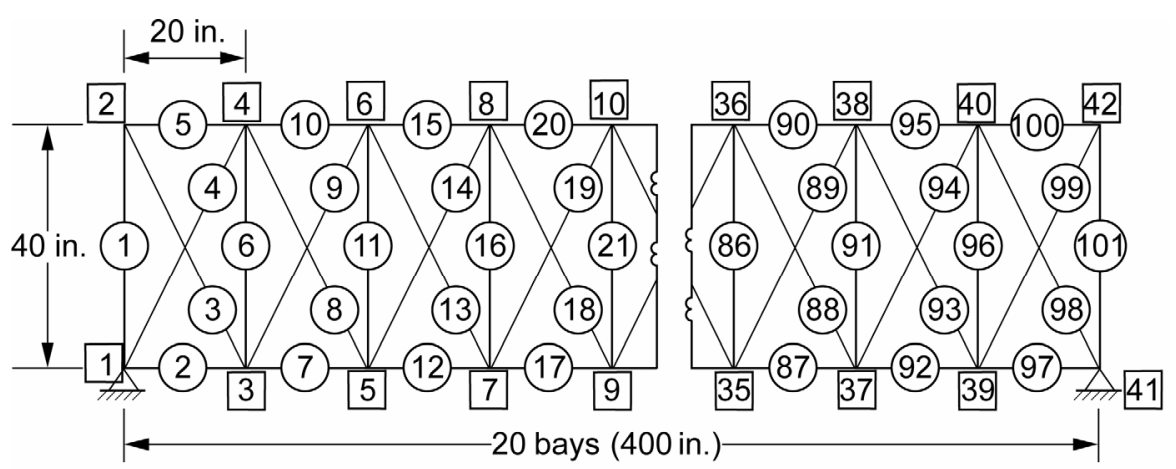

Figure 10.-Twenty-bay truss.

$$
\{A\}^{A_{2} \text { and } A_{3}}=\left\{\begin{array}{l}
0.50 \\
0.71 \\
0.71
\end{array}\right\} \text { in. }^{2} \text { and weight }=63.8 \mathrm{lbf}
$$

The weight of the structure is increased to 71.5 or $63.8 \mathrm{lbf}$ from the weight of $43.4 \mathrm{lbf}$ for the determinate system, when areas are linked. Furthermore, the weight of the structure depends on the choice of the link strategy. For the three-bar truss, weight was increased by 65 percent from the determinate truss when areas $A_{1}$ and $A_{3}$ were linked. The increase was 47 percent for the link condition $A_{2}=A_{3}$.

The link strategy has considerable influence on the weight of the structure. However, it is not straightforward to determine the best link strategy. The bandwidth of the CC provides some analytical guidance to develop a link strategy. Consider for example the 20-bay truss shown in figure 10 . This truss has 101 members with 21 redundancies and 21 CCs. The bandwidth of the CCs are 6 for 20 of them and 20 for 1 of them. The bandwidth of six pertains to a bay in the truss; that is, the first bay has six bars (1 to 6). The areas of these six bars are candidates for linking. A simple strategy is to link the diagonal bars $\left(A_{3}=A_{4}\right)$. Likewise, link bars $\left(A_{8}\right.$ $\left.=A_{9}, A_{13}=A_{14}, \ldots, A_{98}=A_{99}\right)$ for other bays. The remaining CC pertains to the 20 bars of the bottom chord. A simple strategy is to link all such bars $\left(A_{2}=A_{7}=A_{12}=\ldots A_{97}\right)$. The compatibility bandwidth provided a guideline, but there is no unique strategy. It may be tempting to employ linear programming for the purpose. The stiffness method cannot provide a link strategy because of the nonavailability of the bandwidth of the CCs.

The CC explained why an indeterminate structure cannot be fully stressed. The bandwidth of the $\mathrm{CC}$ can be used to develop a linking strategy.

\subsection{Design for Stiffness}

Design for stiffness included displacement limitations in addition to the strength constraints. The design obtained for strength remained adequate if displacement became a passive constraint. The design has to be modified when displacement constraints become infeasible. The design can be prorated uniformly to satisfy the displacement limitations. The prorated technique can produce an overdesign condition because of an underutilization of the strength. The following discussion is an attempt to alleviate the overdesign condition.

4.2.1 Stress-displacement relationship.-Displacement and stress are dependent upon one another, regardless of the bar area of a truss. The dependency relationship (ref. 29) is derived for the three-bar truss shown in figure 5.

$$
\begin{gathered}
\{\beta\}=[G]\{F\}=[B]^{T}\{X\} \\
\sigma_{1}=\frac{E}{2 a}\left(X_{1}-X_{2}\right) \\
\sigma_{2}=-\frac{E}{2 a}\left(X_{2}\right) \\
\sigma_{3}=-\frac{E}{2 a}\left(X_{1}+X_{2}\right) \\
X_{1}=-\frac{a}{E}\left(\sigma_{1}-\sigma_{3}\right) \\
X_{2}=-\frac{a}{E}\left(\sigma_{2}\right)
\end{gathered}
$$

The displacement and stress relationship can be generalized in the following formula:

$$
\sigma_{k}=\sum_{j=p_{1}}^{p_{4}} c_{j k} X_{j}
$$

The coefficients $c_{j k}$ are independent of the design variables, here bar areas. The actual number of displacement components $X_{j}$ in equation (61) depends on the column bandwidth of the equilibrium matrix $[B]$ that is a small number. The maximum bandwidth is four for a 
two-dimensional truss. It is six for a space truss. In other words, a small number of displacements are related to a stress component. For the three-bar truss, displacement depended on one or two bar stresses.

One approach is to modify the stress allowable or strength for selected members using equation (61). Then proceed with the fully stressed design. Let us assume the design for strength obtained for the three-bar truss indicated a 15 percent violation on the limitation specified on the displacement component $X_{2}$. From equation (60f), we can satisfy the displacement constraint if the corresponding allowable stress is reduced by 15 percent $\left(\sigma_{20}=0.85 \sigma_{0}\right)$. This in turn would increase the area of second bar by 17.6 percent $\left(A_{2}^{\text {new }}=1.176 A_{2}^{\text {old }}\right)$. The design update requires no iteration for a determinate truss. Iteration may be necessary for an indeterminate truss. The rule is easily generalized for a truss with $k$ violated displacements.

4.2.2 Design formula for displacement limitation.-A general formula to design for displacement limitation can be obtained by modifying the displacement-force relationship of the IFM.

$$
\{X\}=[J][G]\{F\}
$$

The rectangular matrix $\left[J\right.$, is replaced by $\left[[S]^{-1}\right]^{T}$ with appropriate modification to obtain a displacement formula as follows:

$$
\left\{\frac{\{X\}}{\{0\}}\right\}=\left[[S]^{-1}\right]^{T}[G]\{F\}=[\bar{S}]\{A\}
$$

Equation (63) is manipulated to obtain an expression for the bar areas for displacement limitations as

$$
[\bar{S}]\{A\}=\left\{\frac{\{X\}}{\{0\}}\right\} \text {, or }\{A\}=[\bar{S}]^{-1}\{\bar{X}\}
$$

Equation (64) is combined with equation (58) to obtain formulae for stress and displacement limitations:

$$
\begin{gathered}
\{A\}^{\text {stress }}=\operatorname{abs}\left(\left(\frac{1}{\sigma_{0}}\right)[S]^{-1}\{P\}\right) \\
\{A\}^{\text {disp }}=\operatorname{abs}\left([\bar{S}]^{-1}\{\bar{X}\}\right) \\
\{A\}=\max \left(\{A\}^{\text {stress }},\{A\}^{\text {disp }}\right)
\end{gathered}
$$

The formulae in equation (65) are modified for computational efficiency and superior convergence characteristics to obtain a method called modified fully utilized design (MFUD). Discussion of the MFUD technique is given in reference 31 and is not repeated here. However, results for a

\begin{tabular}{|c|c|c|c|c|c|c|}
\hline \multirow[t]{3}{*}{ Problem } & \multicolumn{2}{|c|}{$\begin{array}{c}\text { Normalized } \\
\text { weight }^{\mathrm{a}}\end{array}$} & \multicolumn{4}{|c|}{ Number of active constraints } \\
\hline & \multirow[t]{2}{*}{ SUMT $^{b}$} & \multirow[t]{2}{*}{ MFUD $^{\mathrm{c}}$} & \multicolumn{2}{|c|}{ Stress } & \multicolumn{2}{|c|}{ Displacement } \\
\hline & & & SUMT & MFUD & SUMT & MFUD \\
\hline 3-bar truss & 1.0 & 1.00 & 2 & 2 & 1 & 1 \\
\hline 5-bar truss & 1.0 & 1.00 & -- & -- & 1 & 1 \\
\hline $\begin{array}{l}\text { Tapered 5-bar } \\
\text { truss }\end{array}$ & 1.0 & 1.00 & 2 & 3 & 1 & 2 \\
\hline 10-bar truss & 1.0 & 1.02 & 1 & -- & 2 & 2 \\
\hline $\begin{array}{l}\text { Tapered 10- } \\
\text { bar truss }\end{array}$ & 1.0 & 1.00 & 5 & 3 & 2 & 2 \\
\hline 25-bar truss & 1.0 & 1.00 & 2 & 4 & 4 & 4 \\
\hline 20-bay truss & 1.0 & 1.02 & 13 & 13 & 2 & 2 \\
\hline $\begin{array}{l}\text { 60-bar trussed } \\
\text { ring }\end{array}$ & 1.0 & 1.00 & 11 & 18 & 1 & 1 \\
\hline $\begin{array}{l}\text { Geodesic } \\
\text { dome }\end{array}$ & 1.0 & 1.01 & 46 & 46 & 1 & 1 \\
\hline
\end{tabular}
set of nine problems are presented in table IV. Each problem is solved twice: first by a design optimization method and then by MFUD.

TABLE IV.-NORMALIZED WEIGHT OBTAINED BY SUMT AND MFUD METHODS

${ }^{\mathrm{a}}$ Weight is normalized with respect to SUMT solution.

${ }^{\mathrm{b}} \mathrm{SUMT}$ is sequential unconstrained minimization technique.

${ }^{c}$ MFUD is modified fully utilized design.

Consider for example the design optimization of the 60-bar trussed ring. It was subjected to three load cases. It had 60 stress and 6 displacement constraints for each load case. The design problem was solved using MFUD and an optimization method using sequential unconstrained minimization technique (SUMT). The 60 bar areas were linked to obtain 25 design variables. For the MFUD method and SUMT algorithm, the optimum weights were 308.1 and $309 \mathrm{lbf}$, respectively. The active constraint set for MFUD method included 18 stresses and 1 displacement, against 11 stresses and 1 displacement for SUMT. The MFUD method generated a good design solution with few calculations. The other eight examples in table IV follow the pattern of the ring problem with minor variations.

\subsection{Design Optimization}

Because of dependent constraints, an optimization algorithm can encounter difficulty in reaching an optimal solution for an indeterminate structural design problem. For example, in an $n$-bar truss, $r$ out of $n$ bar stresses $\{\sigma\}$ are dependent. Stresses are dependent because of the $r$ compatibility conditions, which can be written as $[\bar{C}]\{\sigma\}=\{0\}$.

The $r \times n$ matrix $[\bar{C}]$ is independent of the design variables. Likewise stresses and displacements are dependent. The 
$m=n-r$ number of displacements $\{X\}$ are dependent on the $n$ bar stresses: $\{X\}=[\bar{B}]^{T}\{\sigma\}$. Again the $m \times n$ matrix $[\bar{B}]$ is independent of the design variables.

In an optimization algorithm, the calculation of the search direction $\{d\}$ requires the constraint gradient matrix $[\nabla g]$. An example of the use of the sensitivity matrix to generate the direction follows:

$$
\{d\}=[Q]\{\nabla \mathrm{f}\}
$$

where $[Q]=\frac{[I]-[\nabla g]\left[[\nabla g]^{T}[\nabla g]\right]^{-1}[\nabla g]^{T}}{0.5 \sqrt{\{[H]\{\nabla \mathrm{f}\}\}^{T}\{[H]\{\nabla \mathrm{f}\}\}}}$
$[H]=[I]-[\nabla g]\left[[\nabla g]^{T}[\nabla g]\right]^{-1}[\nabla g]^{T}$

$\mathrm{f}$ is the objective function

The constraint dependency makes the matrix $\left[[\nabla g]^{T}[\nabla g]\right]$ singular. An optimization algorithm may yield a solution despite the singularity condition because most often the $[Q]$ matrix is approximated; it is seldom calculated in closed form. It is reinitialized into an identity matrix when corruption is suspected. Two approaches have been devised to alleviate the singularity issue in optimization. The independent constraints can be segregated (ref. 30), or the design sensitivity can be approximated (ref. 32).

4.3.1 Segregation of independent constraints. - The segregation of a set of independent constraints from all the specified set of constraints is straightforward though it adds extra calculations to optimization, which is already computationally intensive. The process is illustrated considering the three-bar truss as an example, see figure 9(b). The problem has three design variables and five constraints. The constraint gradient or the design sensitivity matrix can be arranged as

$$
\begin{aligned}
& {\left[\left\{\nabla \sigma_{1}\right\}\left\{\nabla \sigma_{2}\right\}\left\{\nabla \sigma_{3}\right\}\left\{\nabla X_{1}\right\}\left\{\nabla X_{2}\right\}\right]} \\
& \stackrel{\text { reduce to }}{\Rightarrow}[\overbrace{\left\{\nabla \sigma_{1}\right\}\left\{\nabla X_{2}\right\}}^{\text {independent }}: \overbrace{\left\{\nabla \sigma_{2}\right\}\left\{\nabla X_{1}\right\}\left\{\nabla \sigma_{3}\right\}}^{\text {dependent }}]
\end{aligned}
$$

A lower and upper triangular factorization can separate the two independent constraint gradients $\nabla \sigma_{1}$ and $\nabla X_{2}$, which can be used to calculate the search direction. The factorization process has to be repeated before the calculation of each
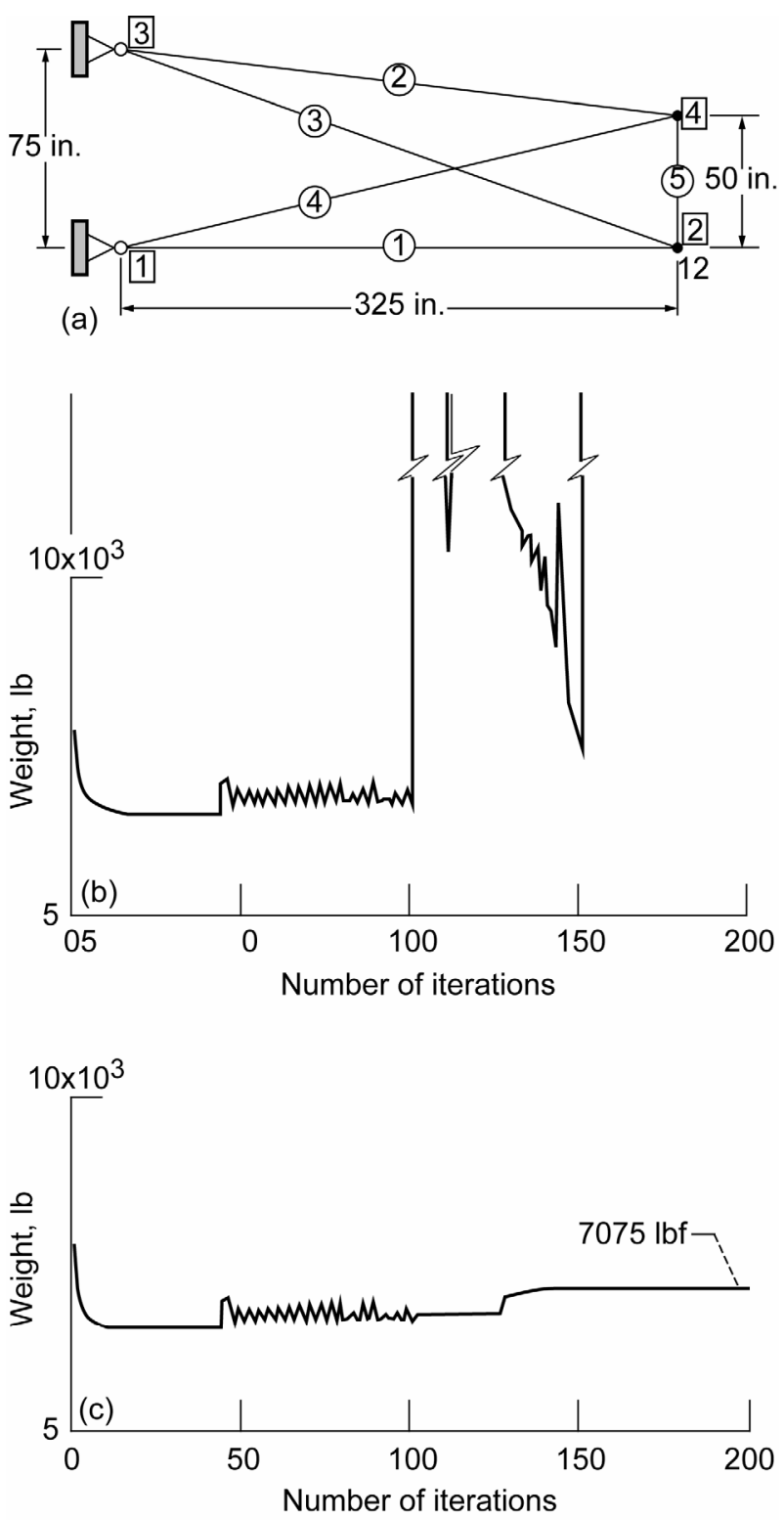

Figure 11.-Design optimization of five-bar truss.

(a) Tapered one-bay truss. (b) Standard optimization.

(c) Optimization accounting for singularity.

search direction because the values of the gradients depend on the current values of the design variables.

The singularity issue is illustrated considering the design optimization of the five-bar truss shown in figure 11(a). Optimum solution is to be obtained for the minimum weight condition for stress and displacement constraints. A quadratic programming algorithm was used to solve the problem. The design solutions are graphed in figures 11(b) and (c) for two cases: 
(1) Singularity is disregarded (see fig. 11(b)).

(2) Singularity is alleviated through a singular value decomposition (see fig. 11(c)).

Design case 1, for which independent constraints were not separated, exhibited a wide variation in the weight, and it failed to converge. Case 2, which used independent constraints, converged to the optimum solution.

4.3.2 Approximate sensitivity.-Design sensitivity can be approximated for an indeterminate structure. The approximation alleviates the singularity condition. It also substantially reduces the number of calculations in structural optimization. The sensitivity matrix for the stress and displacement constraints for an $n$-bar truss with $r$ redundant members is obtained by differentiating the IFM equations (ref. 33). Consider the closed-form sensitivity matrix for stress. It has two distinct factors as follows:

$$
\begin{aligned}
& {[\nabla \sigma]=\left[\nabla \sigma_{1}, \nabla \sigma_{2}, \ldots, \nabla \sigma_{n}\right]}
\end{aligned}
$$

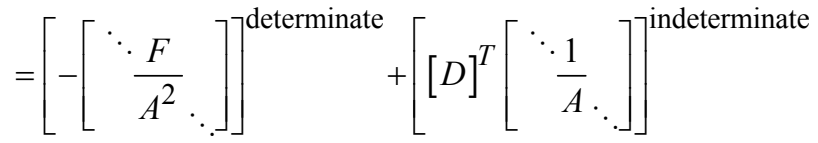

The first factor with superscript "determinate" has to be used for both determinate and indeterminate trusses. The second factor with superscript "indeterminate" is applicable only to indeterminate structures.

The rank of the $n \times n$ sensitivity matrix $[\nabla \sigma]$ is reduced to $m=n-r$. However, it has full rank $n$ for the first term with superscript "determinate." It is also a diagonal matrix and requires a trivial amount of calculation. The second term, superscripted "indeterminate," is responsible for reducing the rank, and its generation is computationally intensive. The proposition is to use only the first term in equation (68).

The approximation process can be extended to the displacement constraint to obtain

$$
[\nabla X]=\left[\begin{array}{l}
{\left[[J]\left[\because S_{\mathrm{dg}} \cdot\right]\right]^{\text {determinate }}} \\
+[[J][G][D]]^{\text {indeterminate }}
\end{array}\right]^{T}
$$

The proposition is to retain the first term in the displacement sensitivity. The sensitivity expressions given in equations (68) and (69) should be adjusted for the allowable limits prior to their use in design optimization. The definitions of the symbols in equations (68) and (69) are as follows:

$$
[D]=[S]^{-1}\left[\begin{array}{l}
{[0]} \\
-[C][\overline{\bar{E}} \\
{[C]}
\end{array}\right][\bar{F}]
$$

The elements of the diagonal matrix $\left[{ }^{\ddots} S_{\mathrm{dg}} \cdot\right]$ are given by

$$
\left(S_{\mathrm{dg}}\right)_{i i}=-\frac{g_{i i} F_{i}}{A_{i}}=-\frac{\ell_{i} F_{i}}{A_{i}^{2} E_{i}}
$$

The diagonal matrices $\left[\begin{array}{l}\ddots \\ \left.\frac{1}{A} \cdot\right] \\ \end{array}\right.$ and $\left[{ }^{\ddots} \cdot \bar{F} \cdot\right]$ are of dimensions $n \times n$, and their elements are $\frac{1}{A_{i}}, \frac{F_{i}}{A_{i}^{2}}, \frac{\ell_{i}}{A_{i}^{2} E_{i}}$, and $F_{i}$, respectively.

The determinate factor in the sensitivity of displacement can be specialized for an $n$-bar indeterminate truss as

$[\nabla X]^{\text {determinate }}=\left[[J]\left[S_{\mathrm{dg}}\right]\right]^{T}=\left(\begin{array}{lll}\ddots & & 0 \\ & \frac{-F \ell}{A^{2} E} & \\ 0 & & \ddots\end{array}\right)[J]^{T}$

Calculation of the determinate sensitivity for the displacement essentially requires a back-substitution step with the factored form of the inverse of the $[S]$ matrix. The inverse matrix calculated to generate force is available at this stage.

Sensitivities of both the stress and displacement contain the member forces $\{F\}$ and the square of bar areas $\{A\}$. Even for an active displacement constraint, the design in essence is modified through the member force. The geometric parameters and material properties of the truss are not explicitly contained in the sensitivity expression for stress because it is a local variable. Because displacement is a global variable, its sensitivity expression explicitly contains the configuration parameters, the material properties, and the design variables.

The proposed approximate sensitivity of stress and displacement constraints alleviates the singularity condition in the design optimization of an indeterminate truss because the coefficient matrix in the determinate factor (see eqs. (68) 
TABLE V.-OPTIMUM SOLUTION FOR 20-BAY TRUSS DESIGN PROBLEM SHOWN IN FIGURE 10

\begin{tabular}{|l|c|c|c|c|c|c|c|c|c|}
\hline Sensitivity & Weight, & \multicolumn{4}{|c|}{$\begin{array}{c}\text { Design variables, } \\
\text { bar area in in. }\end{array}$} & \multicolumn{3}{|c|}{ Active constraints } & \multirow{2}{*}{$\begin{array}{c}\text { Computation time, } \\
\text { sec }\end{array}$} \\
\cline { 3 - 9 } & & $A_{1}$ & $A_{2}$ & $A_{3}$ & $A_{4}$ & $A_{5}$ & Stress & Displacement & \\
\hline Simple & 2023.2 & 3.44 & 7.03 & 0.41 & 1.38 & 1.38 & 3 & 1 & 2.5 \\
\hline Analytical & 2021.8 & 3.44 & 6.99 & 0.41 & 1.39 & 1.39 & 3 & 1 & 14.8 \\
\hline
\end{tabular}

and (69)) has a full rank. The optimum solution is reached with fewer calculations because the optimization process becomes more robust and the sensitivity is generated with a trivial amount of computations. The benefit that accrues from the use of approximate sensitivity is discussed in reference 32. Here, the performance of approximate sensitivity is illustrated considering the 20-bay truss shown in figure 10 as an example. The truss was subjected to three load cases. The first load case consisted of forces in the negative y-coordinate direction along the bottom chord nodes: -40 kip at the midspan and -1 kip at the other nodes. For the second load case, all the top chord nodes were subjected to a 3-kip force along the $x$-coordinate direction. For the third load case, all the bottom chord nodes were subjected to a -3-kip force along the negative $x$-coordinate direction. The allowable stress was $\sigma_{0}=20 \mathrm{ksi}$. A displacement limitation of $0.5 \mathrm{in}$. was imposed at the midspan nodes 21 and 22 along the $x$ - and $y$-coordinate directions, respectively. The $101 \mathrm{bar}$ areas were grouped to obtain five linked design variables. The first two design variables $A_{1}$ and $A_{2}$ represented the bar area of the top and bottom chord members, respectively. All 21 vertical bar areas were grouped to obtain the third variable $A_{3}$. The last two design variables $A_{4}$ and $A_{5}$ represented the bar area of the leading and lagging diagonal members, respectively. The five-variable problem had a total of 312 stress and displacement constraints. Optimum solutions obtained by the sequential quadratic programming (SQP) algorithm are given in table $\mathrm{V}$. The convergence of weight versus the computation time to solution is graphed in figure 12. The convergence patterns for both the closed-form and approximate sensitivities graphed in figure 12 display

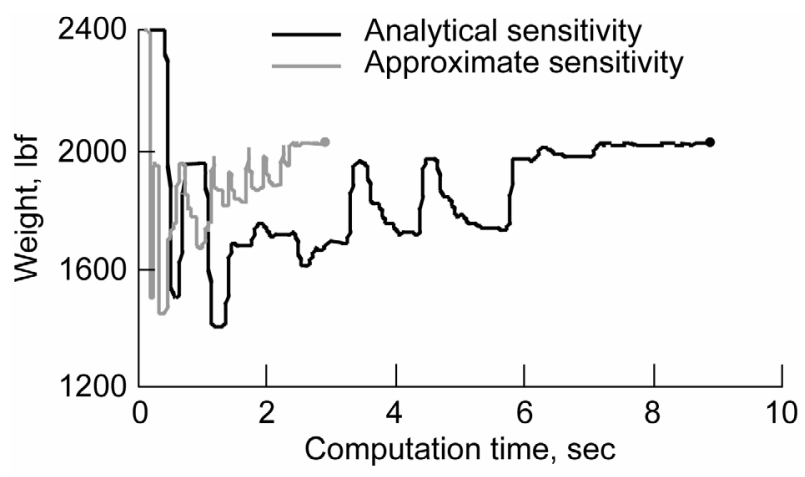

Figure 12.-Solution time for sequential quadratic programming (SQP) algorithm for 20-bay truss. similar undulations. The SQP algorithm converged to the same solution for the determinate as well as for the analytical sensitivities. However, calculations with approximate sensitivities converged 590 percent faster than those with the closed-form gradients. The approximate sensitivity outperformed the analytical gradients in the design optimization problem for the truss.

\subsection{Summary on Design}

The compatibility condition considerably influences the design of an indeterminate structure because design is stress driven. Ignoring compatibility amounts to a brute-force approach that is not likely to produce a robust design that industry would use. The infeasibility of fully stressed design, singularity in optimization, and sensitivity calculations in structural design have been examined for truss-type structures. The same three issues should be examined for flexural members following the approach that has been established for the truss. The behavior study for flexural members is more difficult because stress varies along the member length and depth. Understanding the role of compatibility will provide an insight to behavior of flexural members, leading to a more robust design method. The neglected fully stressed (or utilized) design concept should be revived through use of the compatibility conditions because it has the potential to become an alternate tool for the design optimization method. It is simple and is practiced in industry. We have addressed modified fully utilized design (MFUD) in the stress and displacement constraints for truss design. From solution of many design problems, it is observed that properly formulated MFUD can match or quite often exceed the performance of optimization methods. MFUD should be extended to flexural members beginning with continuous beams and then frames with a variable depth member.

\subsection{Concluding Remarks}

The completed Beltrami-Michell formulation (CBMF), which is a tensorial approach in elasticity (with stress as its primary unknown), is presented. The tensorial method places simultaneous emphasis on the equilibrium equation and the compatibility condition. The displacement method and mixed method as well as the total formulation can be obtained from this method as specialized cases. Such methods become equivalent yielding identical solutions. Fidelity of the stress solution can be guaranteed when it is generated via the CBMF or methods derived from the CBMF. 
A displacement method that is not derived from the CBMF but formulated independently from a consideration of the equilibrium equation should be examined for a compliance of the compatibility condition in the field and on the boundary.

The observations for the CBMF apply to the integrated force method (IFM) for discrete analysis because the internal force unknowns being stress resultants retain the seed of a tensor. That is, the IFM becomes a tensor type of method that emphasizes both stress equilibrium and strain compatibility to yield high-fidelity response even for a modest finite element model. The derived dual integrated force method (IFMD) displacement formulation, being equivalent to the IFM, retained the strength of the IFM. The IFMD should replace the popular stiffness method especially because a code based on the existing stiffness method can be changed to an IFMD code with a small amount of programming effort.

The equations of the IFM were used to formulate a fully utilized design method for stress and displacement constraints. The compatibility condition provides an insight to the behavior of an indeterminate structure, like the infeasibility of full stress design. It identified singularity in structural optimization, simplified the gradient of behavior constraints, alleviated singularity in structural optimization, and reduced computation time to solution.
The tensorial approach could not be developed earlier because the compatibility condition was not fully understood either in elasticity or in structures. The strain formulation cannot be ignored because the compatibility concept makes solid mechanics a research discipline that is practiced in academia and large research organizations throughout the world. Without the compatibility concept, the solid mechanics discipline would degenerate into a few determinate analysis courses in applied mathematics. The use of a fully developed strain formulation has allowed a free movement between different analysis methods and response variables (like force (stress) and displacement). High-speed computers are extremely helpful but cannot replace the strain formulation in elasticity and the compatibility conditions in structural mechanics. The vacuum created when the researchers knowledgeable in strain formulation retire may be difficult to fill. The premier academic and research institutions should encourage the study of the compatibility condition for both linear and nonlinear problems thereby completing the theory of the solid mechanics discipline.

National Aeronautics and Space Administration John H. Glenn Research Center at Lewis Field Cleveland, Ohio 44135, May 14, 2007 



\section{Appendix A \\ Symbols and Acronyms}

$\begin{array}{ll}\text { Symbols } & \\ A & \text { strain energy } \\ B & \text { complementary strain energy } \\ b & \text { body force } \\ {[B]} & \text { equilibrium matrix } \\ {[C]} & \text { compatibility matrix } \\ D & \text { elastic domain } \\ {[D]} & \text { governing matrix of IFMD } \\ \{d\} & \text { search direction } \\ E & \text { Young's modulus } \\ F_{i} & \text { component of }\{F\} \\ \{F\} & \text { internal force vector } \\ \mathrm{f} & \text { objective function } \\ f_{c} & \text { strain formulation function } \\ {[\mathrm{G}]} & \text { flexibility matrix } \\ G_{r}, G_{\theta} & \text { coefficient functions in Green's theorem } \\ {[\nabla g]} & \text { sensitivity matrix of constraints } \\ {[J]} & \text { displacement coefficient matrix } \\ {[K]} & \text { stiffness matrix } \\ \ell & \text { length of boundary } \\ \ell_{1} & \text { length of boundary segment } 1 \\ \ell_{2} & \text { length of boundary segment } 2 \\ M_{x}, M_{y}, M_{x y} & \text { moments } \\ m & \text { number of displacement unknowns } \\ {[M]} & \text { mass matrix } \\ n & \text { number of force unknowns } \\ n_{x}, n_{y}, n_{z} & \text { direction cosines of outward normal } \\ P & \text { load } \\ \{P\} & \text { load vector } \\ \bar{P}_{r}, \bar{P}_{\theta} & \text { external prescribed loads in } r \text { and } \theta \text { directions } \\ p_{i} & \text { respectively } \\ \left\{P^{\text {stiff }}\right\} & \text { traction component } \\ q & \text { stiffness method load vector } \\ R & \text { transverse load } \\ \{\delta R\} & \text { reaction } \\ r, \theta & \text { initial deformation vector } \\ r & \text { polar coordinates } \\ & \text { number of redundant force parameters } \\ & \end{array}$

$\begin{array}{ll}{[S]} & \text { governing matrix of IFM } \\ T & \text { temperature } \\ t & \text { plate thickness } \\ u, v & \text { in-plane displacement } \\ V & \text { body force potential } \\ W & \text { potential of work done } \\ w & \text { transverse displacement } \\ X & \text { component of }\{X\} \\ \{X\} & \text { displacement vector } \\ x, y & \text { Cartesian coordinates } \\ \alpha & \text { coefficient of thermal expansion } \\ \{\beta\} & \text { deformation vector } \\ \left\{\beta^{0}\right\} & \text { initial deformation vector } \\ \gamma & \text { shear strain } \\ \varepsilon_{i j} & \text { strain tensor } \\ {[\kappa]} & \text { material matrix } \\ \pi_{s} & \text { variational functional of IFM } \\ \sigma & \text { stress } \\ \sigma_{0} & \text { yield stress } \\ {[\nabla \sigma]} & \text { sensitivity matrix for stress } \\ \tau & \text { shear stress } \\ \tau_{i j} & \text { stress tensor } \\ v & \text { Poisson's ratio } \\ \varphi & \text { stress function } \\ \omega & \text { circular frequency }\end{array}$

\section{Acronyms}

BCC boundary compatibility condition CBMF completed Beltrami-Michell formulation CC compatibility condition DDR deformation-displacement relation EE equilibrium equation FSD fully stressed design IFM integrated force method IFMD dual integrated force method MFUD modified fully utilized design SQP sequential quadratic programming SUMT sequential unconstrained minimization technique 



\section{Appendix B \\ Further Reading on the Method of Force}

Hellinger, E.: Die allgemeinen Ansätze der Mechanik der Kontinua. Encyklopädia der Mathematischen Wissenchaften, vol. 4, F. Klein and C. Müller, eds., Druck und Verlag von B.G. Teubner, Leipzig, Germany, 1914, pp. 602-694.

Dayaratnam, P.; and Patnaik, S.: Feasibility of Full Stress Design. AIAA J., vol. 7, no. 4, 1969, pp. 773-774.

Patnaik, S.; and Dayaratnam, P.: Behaviour and Design of Pin Connected Structures. Int. J. Numer. Methods Eng., vol. 2, no. 4, 1970, pp. 579-595.

Patnaik, S.: An Integrated Force Method for Discrete Analysis. Int. J. Numer. Methods Eng., vol. 6, no. 2, 1973, pp. 237-251.

Patnaik, S.N.; and Srivastava, N.K.: On Automated Optimum Design of Trusses. Int. J. Comp. Meth. Applied Mech. Eng., vol. 19, no. 3, 1976, pp. 245-265.

Patnaik, S.N.; and Yadagiri, S.: Design for Frequency by the Integrated Force Method. Int. J. Comp. Meth. Applied Mech. Eng., vol. 16, no. 2, 1978, pp. 213-230.

Patnaik, S.N.; and Srivastava, N.K.: Safety of Optimally Designed Structures Like Cylinders and Plates. Comput. Struct., vol. 11, no. 4, 1980, pp. 363-367.

Patnaik, S.N.; and Yadagiri, S.: Frequency Analysis of Structures by Integrated Force Method. J. Sound Vib., vol. 83, no. 1, 1982, pp. 93-109.

Patnaik, S.N.; and Joseph, K.T.: Compatibility Conditions From Deformation Displacement Relations. AIAA J., vol. 23, no. 8, 1985, pp. 1291-1293.

Patnaik, S.N.: The Variational Energy Formulation for the Integrated Force Method. AIAA J., vol. 24, no. 1, 1986, pp. 129-137.

Patnaik, S.N.: The Integrated Force Method Versus the Standard Force Method. Comp. Struct., vol. 22, no. 2, 1986, pp. 151-163.

Patnaik, S.N.; and Joseph, K.T.: Generation of the Compatibility Matrix in the Integrated Force Method. Comp. Methods Appl. Mech. Eng., vol. 55, no. 3, 1986, pp. 239-257.

Patnaik, S.N.: Behavior of Trusses With Stress and Displacement Constraints. Comput. Struct., vol. 22, no. 4, 1986, pp. 619-623.

Patnaik, S.N.; and Nagaraj, M.S.: Analysis of Continuum by the Integrated Force Method. Comput. Struct., vol. 26, no. 6, 1987, pp. 889-905.

Patnaik, S.N.; and Gallagher, Richard H.: Gradients of Behaviour Constraints and Reanalysis Via the Integrated Force Method. Int. J. Numer. Methods Eng., vol. 23, no. 12 , 1986, pp. 2205-2212.
Vijayakumar, K.; Krishna Murty, A.V.; and Patnaik, S.N.: A Basis for the Analysis of Solid Continua Using the Integrated Force Method. AIAA J., vol. 26, no. 5, 1988, pp. 628-629.

Patnaik, S.N.: Analytical Initial Design for Structural Optimization Via the Integrated Force Method. Comput. Struct., vol. 33, no. 1, 1989, pp. 265-268.

Patnaik, S.N.; and Satish, H.G.: Analysis of Continuum Using Boundary Compatibility Conditions of Integrated Force Method. Comput. Struct., vol. 34, no. 2, 1990, pp. 287-295.

Nagabhusanam, J.; and Patnaik, S.N.: General-Purpose Program to Generate Compatibility Matrix for the Integrated Force Method. AIAA J., vol. 28, no. 10, 1990, pp. 1836-1842.

Patnaik, S.N.; Berke, L.; and Gallagher R.H.: Compatibility Conditions of Structural Mechanics for Finite Element Analysis. AIAA J., vol. 29, no. 5, 1991, pp. 820-829.

Patnaik, S.N.; Berke, L.; and Gallagher, R.H.: Integrated Force Method Versus Displacement Method for FiniteElement Analysis. Comp. Struct., vol. 38, no. 4, 1991, pp. 377-407.

Patnaik, S.N., et al.: Improved Accuracy for Finite-Element Structural-Analysis Via an Integrated Force Method. Comp. Struct., vol. 45, no. 3, 1992, pp. 521-542.

Patnaik, S.N.; Guptill, J.D.; and Berke, L.: Singularity in Structural Optimization. Int. J. Numer. Methods Eng., vol. 36, no. 6, 1993, pp. 931-944.

Patnaik, S.N.; Hopkins, D.A.; and Coroneos, R.: Structural Optimization With Approximate Sensitivities. Comput. Struct., vol. 58, no. 2, 1996, pp. 407-418.

Patnaik, S.N., et al.: Completed Beltrami-Michell Formulation for Analyzing Mixed Boundary Value Problems in Elasticity. AIAA J., vol. 34, no. 1, 1996, pp. 143-148.

Kaljevic, I.; Patnaik, S.N.; and Hopkins, D.A.: Development of Finite Elements for Two-Dimensional Structural Analysis Using the Integrated Force Method. Comput. Struct., vol. 59, no. 4, 1996, pp. 691-706.

Kaljevic, I.; Patnaik, S.N.; and Hopkins, D.A.: ThreeDimensional Structural Analysis by the Integrated Force Method. Comput. Struct., vol. 58, no. 5, 1996, pp. 869-886.

Kaljevic, Igor; Patnaik, Surya N.; and Hopkins, Dale A.: Treatment of Initial Deformations in the Integrated Force Method. Comput. Meth. Appl. Mech. Eng., vol. 140, nos. 3-4, 1997, pp. 281-289. 
Patnaik, S.N.; Coroneos, R.M.; and Hopkins, D.A.: Dynamic Animation of Stress Modes Via the Integrated Force Method of Structural Analysis. Int. J. Numer. Methods Eng., vol. 40, no. 12, 1997, pp. 2151-2169.

Park, K.C.; and Felippa, C.A.: A Variational Framework for Solution Method Developments in Structural Mechanics. J. Appl. Mech., vol. 65, no. 1, 1998, pp. 242-249.
Reich, G.W.; and Park, K.C.: A Theory for Strain-Based Structural System Identification. J. Appl. Mech., vol. 68, 2001, pp. 521-527.

Grycz, J.M.: On the Conditions of Compatibility for the Plane Stress State and Hooke's Law. O Warunkach Nierozdzielności w Założeniu Plaskiego Stanu Napężenia Struktur Wykonanych z Tworzyw Podlegających Aksjomatowi Hooke'a, Archives of Civil Engineering, vol. L, no. 2, 2004, pp. 191-202. 


\section{Appendix C \\ Solution of Elasticity Problem With BCC}

The use of the boundary compatibility condition (BCC) is illustrated through the solution of an elasticity problem. In addition, the BCCs are listed for a three-dimensional elastic continuum.

\section{C.1 Use of BCC in CBMF}

The use of the BCC in the solution of the completed Beltrami-Michell formulation (CBMF) is illustrated considering the example of the annular plate shown in figure 13. In the text solution for the problem was included for the case of a thermal load. For simplicity the solution strategy in this appendix is illustrated for a mechanical load $(P)$ applied on the outer boundary, at $(r=a)$, as depicted in figure 13. Other parameters of the problem remained unchanged. The field equations and boundary conditions given in the text are adjusted for mechanical load. The traction condition is applied at the outer free boundary, while the BCC is imposed on the inner boundary, which is fixed. The BCC should not be imposed on the outer free boundary.

The equilibrium equation in the field:

$$
\frac{\mathrm{d}}{\mathrm{d} r}\left(\sigma_{r}+\sigma_{\theta}\right)=0
$$

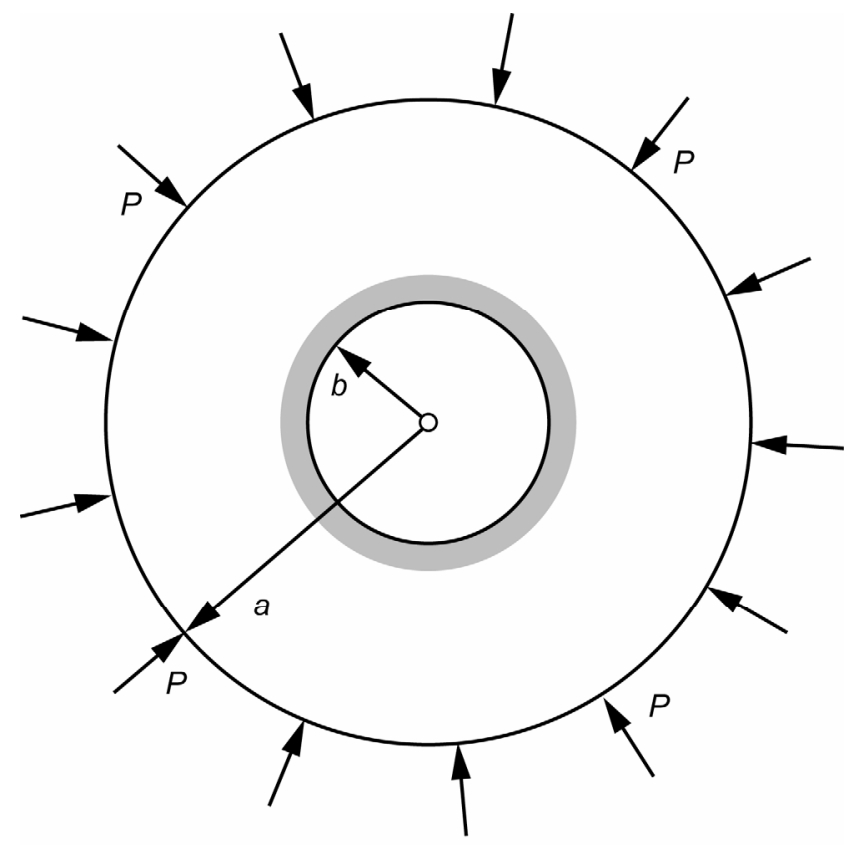

Figure 13.-Annular plate subjected to uniform load $P$.
The field compatibility condition:

$$
\frac{\mathrm{d} \sigma_{r}}{\mathrm{~d} r}+\frac{\left(\sigma_{r}-\sigma_{\theta}\right)}{r}=0
$$

The traction equation along the outer free boundary:

$$
\sigma_{r}=P \text { at } r=a
$$

The boundary compatibility condition along the fixed inner boundary

$$
\sigma_{\theta}-v \sigma_{r}=0 \text { at } r=b
$$

The field equations (eqs. (C1) and (C2)) and the boundary conditions (eqs. (C3) and (C4)) yield the stress solution. Displacement boundary condition is not used to calculate the stress state. Displacement $(u)$ back-calculated from the stress (or strain) contains an integration constant that is evaluated from the prescribed displacement boundary condition imposed along the fixed inner boundary.

$$
u=0 \text { at } r=b
$$

The solution is generated in the following seven steps:

Step 1: The sum of stress $\left(\sigma_{r}+\sigma_{\theta}\right)$ is a constant $\left(c_{0}\right)$ from the field equilibrium equation $(\mathrm{C} 1)$ :

$$
\begin{aligned}
& \sigma_{r}+\sigma_{\theta}=c_{0} \\
& \text { or, } \sigma_{r}=c_{0}-\sigma_{\theta}
\end{aligned}
$$

Step 2: The field compatibility condition equation (C2) is expressed in terms of radial stress $\sigma_{r}$ by eliminating the tangential stress $\sigma_{\theta}$ between equations (C2) and (C6) to obtain an uncoupled differential equation as

$$
\frac{\mathrm{d} \sigma_{r}}{\mathrm{~d} r}+\frac{2}{r} \sigma_{r}=\frac{c_{0}}{r}
$$

Step 3: Equation (C7) is solved to obtain $\sigma_{r}$ as

$$
\sigma_{\theta}=\frac{c_{0}}{2}+\frac{c_{1}}{r^{2}}
$$

Step 4: Stress $\sigma_{\theta}$ is obtained by back substitution in equation (C6) as

$$
\sigma_{r}=\frac{c_{0}}{2}-\frac{c_{1}}{r^{2}}
$$


Step 5: The two constants $\left(c_{0}\right.$ and $\left.c_{1}\right)$ are obtained from the traction and boundary compatibility conditions given by equations (C3) and (C4), respectively.

$$
\begin{aligned}
& \frac{c_{0}}{2}+\frac{c_{1}}{a^{2}}=P \\
& \frac{c_{0}}{2}-\frac{c_{1}}{b^{2}}-v\left(\frac{c_{0}}{2}+\frac{c_{1}}{b^{2}}\right)=0
\end{aligned}
$$

Solution of equation (C10) yielded the two constants:

$$
\begin{aligned}
& c_{0}=\frac{2(1+v) a^{2}}{(1+v) a^{2}+(1-v) b^{2}} P \\
& c_{1}=\frac{(v-1) a^{2} b^{2}}{(1+v) a^{2}+(1-v) b^{2}} P
\end{aligned}
$$

Step 6: The stress solution is obtained from equations (C8), (C9), and (C11) as

$$
\begin{gathered}
\sigma_{r}=\frac{\left[(1+v) r^{2}+(1-v) b^{2}\right]}{(1+v) a^{2}+(1-v) b^{2}}\left(\frac{a^{2}}{r^{2}}\right) P \\
\sigma_{\theta}=\frac{\left[(1+v) r^{2}-(1-v) b^{2}\right]}{(1+v) a^{2}+(1-v) b^{2}}\left(\frac{a^{2}}{r^{2}}\right) P
\end{gathered}
$$

The stress solution is obtained without using the displacement boundary condition.

Step 7: Calculation of displacement involves the transformation of stress to strain. Strain is integrated to obtain displacement. The constant of integration is determined from the displacement boundary condition. Calculation of displacement become trivial for the axis-symmetric annular plate because of the simple strain-displacement relationship.

Stains $\varepsilon_{r}$ and $\varepsilon_{\theta}$ are calculated from stress as

$$
\begin{aligned}
& \varepsilon_{r}=\frac{\left[\left(1-v^{2}\right) r^{2}-\left(1-v^{2}\right) b^{2}\right]}{(1+v) a^{2}+(1-v) b^{2}}\left(\frac{a^{2}}{r^{2}}\right)\left(\frac{P}{E}\right) \\
& \varepsilon_{\theta}=\frac{\left[\left(1-v^{2}\right) r^{2}+\left(1-v^{2}\right) b^{2}\right]}{(1+v) a^{2}+(1-v) b^{2}}\left(\frac{a^{2}}{r^{2}}\right)\left(\frac{P}{E}\right)
\end{aligned}
$$

The displacement can be calculated from the radial strain $\left(\varepsilon_{r}=\frac{\mathrm{d} u}{\mathrm{~d} r}\right)$ or the tangential strain $\left(\varepsilon_{\theta}=\frac{u}{r}\right)$. Both techniques yield the same displacement function, even though one required an integration and determination of the constant from the displacement boundary condition, while the other bypassed such procedure. The displacement function is

$$
u=\frac{\left(1-v^{2}\right)\left(r^{2}+b^{2}\right)}{(1+v) a^{2}+(1-v) b^{2}}\left(\frac{a^{2}}{r}\right)\left(\frac{P}{E}\right)
$$

\section{C.2 Boundary Compatibility Conditions in Three Dimensions}

For a three-dimensional elastic continuum, the BCCs have been derived from the stationary condition of the variational functional of the integrated force method (ref. 34). The variational calculation yielded three field equations (ref. 35) and three boundary compatibility conditions. The BCCs are listed in stress, in strain, and in displacement.

$\mathrm{BCC}$ in stress:

$$
\begin{aligned}
& \frac{\partial}{\partial z}\left\{n_{z}\left(\sigma_{y}-v \sigma_{z}-v \sigma_{x}\right)-n_{y}(1+v) \tau_{y z}\right\} \\
& +\frac{\partial}{\partial y}\left\{n_{y}\left(\sigma_{z}-v \sigma_{x}-v \sigma_{y}\right)-n_{z}(1+v) \tau_{y z}\right\}=0 \\
& \frac{\partial}{\partial x}\left\{n_{x}\left(\sigma_{z}-v \sigma_{x}-v \sigma_{y}\right)-n_{z}(1+v) \tau_{z x}\right\} \\
& +\frac{\partial}{\partial z}\left\{n_{z}\left(\sigma_{x}-v \sigma_{y}-v \sigma_{z}\right)-n_{x}(1+v) \tau_{z x}\right\}=0 \\
& \frac{\partial}{\partial y}\left\{n_{y}\left(\sigma_{x}-v \sigma_{y}-v \sigma_{z}\right)-n_{x}(1+v) \tau_{x y}\right\} \\
& +\frac{\partial}{\partial x}\left\{n_{x}\left(\sigma_{y}-v \sigma_{z}-v \sigma_{x}\right)-n_{y}(1+v) \tau_{x y}\right\}=0
\end{aligned}
$$

BCC in strain:

$$
\begin{aligned}
& \frac{\partial}{\partial z}\left(n_{z} \varepsilon_{y}-\frac{1}{2} n_{y} \gamma_{y z}\right)+\frac{\partial}{\partial y}\left(n_{y} \varepsilon_{z}-\frac{1}{2} n_{z} \gamma_{y z}\right)=0 \\
& \frac{\partial}{\partial z}\left(n_{z} \varepsilon_{x}-\frac{1}{2} n_{x} \gamma_{x z}\right)+\frac{\partial}{\partial x}\left(n_{x} \varepsilon_{z}-\frac{1}{2} n_{z} \gamma_{x z}\right)=0
\end{aligned}
$$

$$
\frac{\partial}{\partial y}\left(n_{y} \varepsilon_{x}-\frac{1}{2} n_{x} \gamma_{x y}\right)+\frac{\partial}{\partial x}\left(n_{x} \varepsilon_{y}-\frac{1}{2} n_{y} \gamma_{x y}\right)=0
$$


BCC in displacement:

$$
\begin{aligned}
& n_{y} \frac{\partial^{2} v}{\partial z^{2}}+n_{z} \frac{\partial^{2} w}{\partial y^{2}}-\frac{\partial^{2}}{\partial y \partial z}\left(n_{z} v+n_{y} w\right)=0 \\
& n_{z} \frac{\partial^{2} w}{\partial x^{2}}+n_{x} \frac{\partial^{2} u}{\partial z^{2}}-\frac{\partial^{2}}{\partial z \partial x}\left(n_{x} w+n_{z} u\right)=0
\end{aligned}
$$

$$
n_{y} \frac{\partial^{2} v}{\partial x^{2}}+n_{x} \frac{\partial^{2} u}{\partial y^{2}}-\frac{\partial^{2}}{\partial x \partial y}\left(n_{y} v+n_{x} u\right)=0
$$

where $\sigma_{x}, \sigma_{y}, \sigma_{z}, \tau_{x y}, \tau_{x z}$, and $\tau_{y z}$ are the stress components; $\varepsilon_{x}$, $\varepsilon_{y}, \varepsilon_{z}, \gamma_{x y}, \gamma_{x z}$, and $\gamma_{y z}$ are the strain components; $u, v$, and $w$ are the displacements; and $n_{x}, n_{y}$, and $n_{z}$ are the direction cosines of the outward normal. The BCCs expressed in terms of continuous displacement functions are not satisfied automatically. 



\section{References}

1. Timoshenko, Stephen: History of Strength of Materials: With a Brief Account of the History of Theory of Elasticity and Theory of Structures. Dover Publications, New York, NY, 1983.

2. Ferrers, N.M., ed.: Mathematical Papers of the Late George Green. Macmillan and Co., London, 1871.

3. Patnaik, S.N.: The Variational Energy Formulation for the Integrated Force Method. AIAA J., vol. 24, no. 1, 1986, pp. 129-137.

4. Przemieniecki, J.S.: Theory of Matrix Structural Analysis. McGraw-Hill, New York, NY, 1968.

5. Patnaik, Surya N.; and Hopkins, Dale A.: Strength of Materials: A Unified Theory for the 21st Century. Elsevier, Amsterdam, 2004.

6. Patnaik, Surya N.; and Hopkins, Dale A.: Completed BeltramiMichell Formulation in Polar Coordinates. NASA/TM-2005213634, 2005.

7. Sokolnikoff, Ivan Stephen: Mathematical Theory of Elasticity. Second ed., McGraw-Hill, New York, NY, 1956.

8. Patnaik, S.N., et al.: Completed Beltrami-Michell Formulation for Analyzing Mixed Boundary Value Problems in Elasticity. AIAA J., vol. 34, no. 1, 1996, pp. 143-148.

9. Patnaik, S.N.; Berke, L.; and Gallagher, R.H.: Integrated Force Method Versus Displacement Method for Finite-Element Analysis. Comp. Struct., vol. 38, no. 4, 1991, pp. 377-407.

10. Patnaik, S.N.: The Integrated Force Method Versus the Standard Force Method. Comp. Struct., vol. 22, no. 2, 1986, pp. 151-163.

11. Patnaik, S.N., et al.: Improved Accuracy for Finite-Element Structural-Analysis Via an Integrated Force Method. Comp. Struct., vol. 45, no. 3, 1992, pp. 521-542.

12. Dayaratnam, P.; and Patnaik, S.: Feasibility of Full Stress Design. AIAA J., vol. 7, no. 4, 1969, pp. 773-774.

13. Patnaik, S.; and Dayaratnam, P.: Behaviour and Design of Pin Connected Structures. Int. J. Numer. Methods Eng., vol. 2, no. 4, 1970, pp. 579-595.

14. Love, A.E.H.: A Treatise on the Mathematical Theory of Elasticity. University Press, Cambridge University Press, England, 1927.

15. Ritz, Walter: Uber eine neue Methode zur Losung Gewisser Variations_-Probleme der Mathematischen Physik. J. Reine Angew Math, Band 135, 1909, pp. 1-61.

16. Timoshenko, Stephen: Theory of Plates and Shells. McGrawHill Book Co., New York, NY, 1940.

17. Park, K.C.; and Felippa, Carlos A.: Variational Principle for the Formulation of Partitioned Structural Systems. Int. J. Numer. Methods Eng., vol. 47, no. 1, 2000, pp. 395-418.

18. Denke, P.H.: A General Digital Computer Analysis of Statically Indeterminate Structures. NASA TN D-1666, 1962.
19. Patnaik, S.N.; and Yadagiri, S.: Frequency Analysis of Structures by Integrated Force Method. J. Sound Vib., vol. 83, no. 1,1982 , pp. 93-109.

20. Patnaik, S.N.; Berke, L.; and Gallagher R.H.: Compatibility Conditions of Structural Mechanics for Finite Element Analysis. AIAA J., vol. 29, no. 5, 1991, pp. 820-829.

21. Hopkins, D., et al.: Fidelity of the Integrated Force Method Solution. Int. J. Numer. Methods Eng., vol. 55, no. 11, 2002, pp. 1367-1371.

22. Patnaik, S.N.; Coroneos, R.M.; and Hopkins, D.A.: Dynamic Animation of Stress Modes Via the Integrated Force Method of Structural Analysis. Int. J. Numer. Methods Eng., vol. 40, no. 12, 1997, pp. 2151-2169.

23. Storaasli, Olaf O.; Baddourah, Majdi; and Nguyen, Duc: GPS: General Purpose Solver. NASA Langley Software, 1995.

24. Harwell Subroutine Library-Specifications. Harwell Laboratory, Oxfordshire, England, 1990.

25. Batoz, Jean-Louis; and Dhatt, G.: Modélisation des Structures par Eléments Finis. Volume 1. Solides Elastiques. Presses de l'Université Laval, Sainte-Foy, 1990.

26. Cilley, Frank H.: The Exact Design of Statically Indeterminate Frameworks-An Exposition of Its Possibility, But Futility. Trans., American Society of Civil Engineers, vol. XLIII, no. 873, 1900, pp. 353-407.

27. Reinschmidt, Kenneth F.; Cornell, C. Allin; and Brotchie, John F.: Iterative Design and Structural Optimization. Journal of Structural Division, Proceedings of the American Society of Civil Engineers, vol. ST 6, 1966, pp. 281-318.

28. Gallagher, Richard H.; and Zienkiewicz, O.C., eds.: Optimum Structural Design. John Wiley \& Sons, London, 1973.

29. Patnaik, S.N.: Behavior of Trusses With Stress and Displacement Constraints. Comput. Struct., vol. 22, no. 4, 1986, pp. 619-623.

30. Patnaik, S.N.; Guptill, J.D.; and Berke, L.: Singularity in Structural Optimization. Int. J. Numer. Methods Eng., vol. 36, no. 6, 1993, pp. 931-944.

31. Patnaik, S.N., et al.: Modified Fully Utilized Design (MFUD) Method for Stress and Displacement Constraints. Int. J. Numer. Methods Eng., vol. 41, no. 7, 1998, 1171-1194.

32. Patnaik, Surya N.; Pai, Shantaram S.; and Hopkins, Dale A.: Precision of Sensitivity in the Design Optimization of Indeterminate Structures. NASA/TP-2006-213818, 2006.

33. Patnaik, S.N.; and Gallagher, Richard H.: Gradients of Behaviour Constraints and Reanalysis Via the Integrated Force Method. Int. J. Numer. Methods Eng., vol. 23, no. 12, 1986, pp. 2205-2212.

34. Patnaik, Surya N.; and Hopkins, Dale A.: Stress Formulation in Three-Dimensional Elasticity. NASA/TP_2001-210515, 2001.

35. Washizu, K.: A Note on the Conditions of Compatibility. J. Math. Phys., vol. 36, no. 2, 1958, pp. 306-312. 


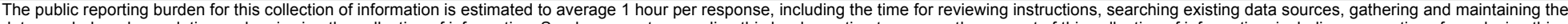

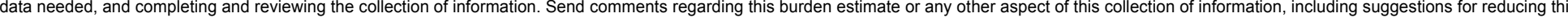

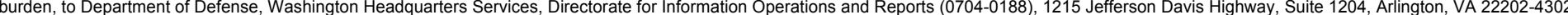

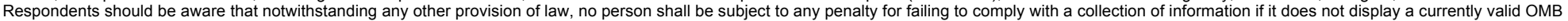
control number.

PLEASE DO NOT RETURN YOUR FORM TO THE ABOVE ADDRESS.

\section{REPORT DATE (DD-MM-YYYY) \\ 2. REPORT TYPE \\ 3. DATES COVERED (From - To)}

14-05-2007

\section{TITLE AND SUBTITLE}

Compatibility Condition in Theory of Solid Mechanics (Elasticity, Structures,

and Design Optimization)

Technical Paper

5a. CONTRACT NUMBER

5b. GRANT NUMBER

NAG3-2915

5c. PROGRAM ELEMENT NUMBER

6. AUTHOR(S)

Patnaik, Surya, N.; Pai, Shantaram, S.; Hopkins, Dale, A.

\section{5d. PROJECT NUMBER}

5e. TASK NUMBER

5f. WORK UNIT NUMBER

WBS 599489.02.07.03.06

\section{PERFORMING ORGANIZATION REPORT NUMBER}

E-15772

National Aeronautics and Space Administration

John H. Glenn Research Center at Lewis Field

Cleveland, Ohio 44135-3191

9. SPONSORING/MONITORING AGENCY NAME(S) AND ADDRESS(ES)

National Aeronautics and Space Administration

Washington, DC 20546-0001

10. SPONSORING/MONITORS
ACRONYM(S)
NASA
11. SPONSORING/MONITORING
REPORT NUMBER
NASA/TP-2007-214480

\section{DISTRIBUTION/AVAILABILITY STATEMENT}

Unclassified-Unlimited

Subject Category: 39

Available electronically at http://gltrs.grc.nasa.gov

This publication is available from the NASA Center for AeroSpace Information, 301-621-0390

\section{SUPPLEMENTARY NOTES}

\section{ABSTRACT}

The strain formulation in elasticity and the compatibility condition in structural mechanics have neither been understood nor have they been utilized. This shortcoming prevented the formulation of a direct method to calculate stress. We have researched and understood the compatibility condition for linear problems in elasticity and in finite element analysis. This has lead to the completion of the "method of force" with stress (or stress resultant) as the primary unknown. The method in elasticity is referred to as the completed Beltrami-Michell formulation (CBMF), and it is the integrated force method (IFM) in structures. The dual integrated force method (IFMD) with displacement as the primary unknown has been formulated. IFM and IFMD produce identical responses. The variational derivation of the CBMF yielded the new boundary compatibility conditions. The CBMF can be used to solve stress, displacement, and mixed boundary value problems. The IFM in structures produced high-fidelity response even with a modest finite element model. The IFM has influenced structural design considerably. A fully utilized design method for strength and stiffness limitation has been developed. The singularity condition in optimization has been identified. The CBMF and IFM tensorial approaches are robust formulations because of simultaneous emphasis on the equilibrium equation and the compatibility condition.

\section{SUBJECT TERMS}

Force method; Indeterminate structure; Boundary compatibility; Design; Singularity

\begin{tabular}{|c|c|c|c|c|}
\hline 16. SECURI & ASSIFICATION & & 17. LIMITATION OF & 18. NUMBER \\
\hline $\begin{array}{l}\text { a. REPORT } \\
U\end{array}$ & $\begin{array}{l}\text { b. ABSTRACT } \\
U\end{array}$ & $\begin{array}{l}\text { c. THIS } \\
\text { PAGE } \\
\text { U }\end{array}$ & & $\begin{array}{c}\text { PAGES } \\
38\end{array}$ \\
\hline
\end{tabular}

19a. NAME OF RESPONSIBLE PERSON
Shantaram S. Pai
19b. TELEPHONE NUMBER (include area code)
216-433-3255



